\title{
Examination of coalescence as the origin of nuclei in hadronic collisions
}

\author{
Francesca Bellini, ${ }^{1,{ }^{*}}$ Kfir Blum, ${ }^{2,3, \dagger}$ Alexander Phillip Kalweit, ${ }^{1,+}$ and Maximiliano Puccio ${ }^{1, \S}$ \\ ${ }^{1}$ Experimental Physics Department, CERN, CH-1211 Geneve 23, Switzerland \\ ${ }^{2}$ Weizmann Institute, Department of Particle Physics and Astrophysics, Rehovot 7610001, Israel \\ ${ }^{3}$ Theoretical Physics Department, CERN, CH-1211 Geneve 23, Switzerland
}

(Received 7 August 2020; accepted 22 December 2020; published 28 January 2021)

\begin{abstract}
The origin of weakly bound nuclear clusters in hadronic collisions is a key question to be addressed by heavy-ion collision (HIC) experiments. The measured yields of clusters are approximately consistent with expectations from phenomenological statistical hadronization models (SHMs), but a theoretical understanding of the dynamics of cluster formation prior to kinetic freeze-out is lacking. The competing model is nuclear coalescence, which attributes cluster formation to the effect of final state interactions (FSI) during the propagation of the nuclei from kinetic freeze-out to the observer. This phenomenon is closely related to the effect of FSI in imprinting femtoscopic correlations between continuum pairs of particles at small relative momentum difference. We give a concise theoretical derivation of the coalescence-correlation relation, predicting nuclear cluster spectra from femtoscopic measurements. We review the fact that coalescence derives from a relativistic Bethe-Salpeter equation, and recall how effective quantum mechanics controls the dynamics of cluster particles that are nonrelativistic in the cluster center-of-mass frame. We demonstrate that the coalescence-correlation relation is roughly consistent with the observed cluster spectra in systems ranging from $\mathrm{PbPb}$ to $p \mathrm{~Pb}$ and $p p$ collisions. Paying special attention to nuclear wave functions, we derive the coalescence prediction for the hypertriton and show that it, too, is roughly consistent with the data. Our work motivates a combined experimental programme addressing femtoscopy and cluster production under a unified framework. Upcoming $p p, p \mathrm{~Pb}$, and peripheral $\mathrm{PbPb}$ data analyzed within such a program could stringently test coalescence as the origin of clusters.
\end{abstract}

DOI: 10.1103/PhysRevC.103.014907

\section{INTRODUCTION}

Loosely bound nuclei like the deuteron (hereafter D), ${ }^{3} \mathrm{He}$, ${ }^{3} \mathrm{H},{ }_{\Lambda}^{3} \mathrm{H}$, and their antiparticles are detected among the products of high-energy hadronic collisions at the CERN Large Hadron Collider (LHC) and other experiments, and their study is a central objective in heavy-ion collision (HIC) experiments [1-3]. Interestingly, the momentum-integrated yields of these nuclei are roughly consistent with being drawn from a thermal distribution with the same temperature parameter $T_{c h}$ that fits the yields of mesons and nucleons [4-7]. Taken together from $\pi^{ \pm}$to ${ }^{4} \mathrm{He}$, the hadron yields span some nine orders of magnitude with only $O(1)$ discrepancies. ${ }^{1}$ This has led some authors to speculate that nuclei take part, on equal

\footnotetext{
*francesca.bellini@cern.ch

${ }^{\dagger}$ kfir.blum@weizmann.ac.il

‡alexander.philipp.kalweit@cern.ch

§maximiliano.puccio@cern.ch

${ }^{1}$ With the discrepancies affecting nuclei [5] at a comparable level to mesons and nucleons [6].
}

Published by the American Physical Society under the terms of the Creative Commons Attribution 4.0 International license. Further distribution of this work must maintain attribution to the author(s) and the published article's title, journal citation, and DOI. footing with the "more fundamental" mesons and nucleons, in an equilibrium partition function characterizing the high excitation state (HXS) produced in HICs. A recent account of this statistical hadronization model (SHM) is given in Ref. [4].

While the SHM is approximately consistent with cluster yields, no first-principle theoretical framework as of yet explains the dynamics of cluster formation in the HXS before kinetic freeze-out. ${ }^{2}$ Clusters are big (several fm) fragile (binding energies $E_{b} \lesssim 10 \mathrm{MeV}$, as low as $E_{b} \approx 0.13 \mathrm{MeV}$ for ${ }_{\Lambda}^{3} \mathrm{H}$ [10]) objects, while even at kinetic freeze-out the HXS does not exceed a few ${ }^{3} \mathrm{fm}$ and it is a hot state with characteristic particle excitation energies of the order of $100 \mathrm{MeV}$. What does it mean for a D with diameter of about $4 \mathrm{fm}$ to exist in an equilibrium distribution in an HXS of diameter $2 \mathrm{fm}$, produced in $p p$ collisions? What does it mean for ${ }_{\Lambda}^{3} \mathrm{H}$, with effective diameter around $14 \mathrm{fm}$ ? This puzzle makes the origin of nuclei uniquely interesting.

\footnotetext{
${ }^{2} \mathrm{~A}$ recent speculation is the formation of compact "preclusters" $[8,9]$ due to an in-medium modification of the nuclear potential.

${ }^{3}$ By this we mainly have in mind the HXS homogeneity radius as revealed by femtoscopy $[11,12]$, to be discussed later on. But even the total HXS volume as fitted in the SHM in high multiplicity $\mathrm{PbPb}$ collisions is only of diameter $20 \mathrm{fm}$, shrinking to $2 \mathrm{fm}$ or so in low multiplicity $p p$ and $p \mathrm{~Pb}$ collisions [6].
} 
The kinetic theory analysis of [13], focusing on D formation in high-multiplicity $\mathrm{PbPb}$ collisions, may shed some light on the problem. This analysis demonstrated that, indeed, D observed at the detector must emerge from the kinetic freezeout region and not from the chemical freeze-out region of the HXS, to which the SHM parameter $T_{c h}$ corresponds. However, Ref. [13] treated the D as a point particle and it is far from clear if and how their analysis could be adapted to smaller systems like $p p, p \mathrm{~Pb}$, or peripheral $\mathrm{PbPb}$ collisions. ${ }^{4}$

An alternative explanation for the origin of nuclei, bypassing the limitations of kinetic theory, is proposed by coalescence. The basic assumption of the coalescence model is that the expansion of the HXS leads to kinetic freeze-out with nucleons but — due to their fragility and size — essentially no nuclei. The HXS at kinetic freeze-out can be described by a quantum mechanical (QM) density matrix. Projecting the density matrix onto particle states at the detector gives the observed particle spectra. Final state interactions (FSI) mediated by nuclear scattering and Coulomb photon exchange enter this projection as they affect the propagation of the particles from the HXS to the detector. FSI manifest themselves in two ways:

(1) FSI imprint momentum correlations among pairs of continuum particles. The analysis of this phenomenon is known as femtoscopy. ${ }^{5}$

(2) FSI also admit discrete bound-state multinucleon solutions, namely nuclei. This is nuclear coalescence.

It is important to note that the coalescence model predicts that the yields of nuclei approximately inherit the thermal spectra of their nucleon constituents, up to a dimensionless QM correction factor. In many cases (e.g., low- $p_{t} \mathrm{D}$ and ${ }^{3} \mathrm{He}$ formation in high multiplicity $\mathrm{PbPb}$ collisions) the $\mathrm{QM}$ correction factor is close to unity $[1,15]$. Thus, the approximately thermal yield of nuclei need not point to the nuclei taking part in an equilibrium partition function before kinetic freeze-out. For some nuclei and systems, however, the QM factor is predicted to be much smaller than unity. An example is the production of ${ }_{\Lambda}^{3} \mathrm{H}$ in $p p$ collisions, or (especially at high- $p_{t}$ ) in low multiplicity $\mathrm{PbPb}$ collisions. These systems offer a key discriminator between the coalescence model and the SHM. Our goal in this paper is to study the theoretical prediction of coalescence, compare to available experimental data, and highlight the path to making this test conclusive.

A central feature in this paper is the relation between coalescence and femtoscopic correlations among continuum nucleons. From the perspective of the coalescence model, femtoscopic correlations and nuclei production are closely related. In a clear sense, the successful reconstruction of the imprint of FSI on pair correlations lends credence to the basic framework of coalescence, which deals with the bound state solutions of essentially the same FSI (in different isospin

\footnotetext{
${ }^{4}$ We thank Urs Wiedemann for pointing out this issue during a workshop at CERN.

${ }^{5}$ The correlation study is also often referred to as Hanbury BrownTwiss (HBT) analysis. For a comparative discussion and historical notes, see [14].
}

channels). Moreover, once femtoscopy calibrates the HXS source characteristics, nuclei yields are predicted without free parameters. Over the years the community (experimental and theoretical) developed a habit of considering femtoscopy and nuclei analyses separately, making it cumbersome to combine the information content of the measurements. One of our goals here is to motivate joint experimental analyses of femtoscopy and cluster yields.

The plan of the paper, along with a brief summary, is as follows.

In Sec. II we briefly review the underlying relation between femtoscopy and cluster formation, defining the coalescence/femtoscopy framework. The basic formalism was laid out by Lednicky et al. [16-18]. We provide a quick reduction of this formalism to observationally accessible objects. This is a good starting point for the discussion, because it demonstrates that coalescence arises in a relativistic quantum field theoretic (QFT) calculation. In Sec. II A we show how, subject to two key approximations (the smoothness approximation and the equal-time approximation), the model-independent coalescence-correlation relation between deuteron production and two-proton femtoscopy comes about. The main result here is the manifest relation between the well-known Eq. (14), for femtoscopy, and Eq. (15), for the $\mathrm{D}$ coalescence factor. This relation was derived first in Ref. [19] starting from the QM limit. Our independent derivation here gives another perspective on this result, showing, for example, that it does not require density matrix factorization to apply for its validity. In Sec. II B we review how adding the assumption of density matrix factorization allows one to connect two-particle femtoscopy analyses to single-particle spectra and three-body coalescence. In Sec. II B 2 we derive the coalescence prediction for ${ }_{\Lambda}^{3} \mathrm{H}$ and ${ }^{3} \mathrm{He}$. Equations (30) and (31), or their momentum space versions of Equations (34) and (35), are the most model-independent versions of these coalescence factors we know of.

None of our results in Sec. II rely on the details of the underlying nucleon emission function, or needs to specify a model of the dynamical evolution of the HXS: our results simply connect femtoscopy with cluster yields, and the connection should apply to any self-consistent HXS model. To make contact with measurements, however, we need to specify the two- and three-particle source. We turn to this in Sec. III. We start in Sec. III A by appealing to observational femtoscopy parametrizations of the two-particle source (extended, with some added assumptions, to the three-particle source). Relying on experimental fits allows us to keep the analysis as model independent as we can. While this is not an essential requirement of the framework, we stick in this paper to Gaussian or semi-Gaussian parametrizations. An effort to improve on the precision of the source determination was carried out in Ref. [20]. In Appendices A and B we give some quantitative model-dependent theoretical examples that suggest that an anisotropic [three-dimensional (3D)] Gaussian source parametrization is probably accurate enough for the purpose of testing the origin of clusters via the coalescencecorrelations relation.

The next ingredient needed is the nuclear wave functions. In Sec. III B we derive the coalescence factors 
corresponding to the simplified Gaussian wave functions. The Gaussian wave function is an oversimplification in some cases, but at the cost of an $O(1)$ theoretical error it allows us to derive analytic results for the coalescence factors, summarized by Eqs. (49)-(51). We note that the Gaussian wave functions we consider allow for different cluster length scales for threebody states; for ${ }_{\Lambda}^{3} \mathrm{H}$ this is crucial, as the $p$ - $n$ factor of the wave function is considerably more compact than the $\Lambda$ - $p n$ factor. Equations (49)-(51) also account for the intrinsically anisotropic shape of the two- and three-particle source describing the HXS. As we illustrate in Appendix B, the two-particle source is expected to be truly anisotropic, especially at large $p_{t}$.

In Sec. III C we extend the analysis to more accurate non-Gaussian wave functions. For D we derive an analytic coalescence factor formula that applies to the Hulthen wave function if the underlying two-particle source is approximated as 1D Gaussian. The more realistic 3D two-particle source can be easily accounted for by numerical integration. For ${ }_{\Lambda}^{3} \mathrm{H}$ we consider the recent three-body wave function proposed by [21]. We show that while the theoretically expected wave function is non-Gaussian, exhibiting an extended high- $q$ tail, nevertheless an effective Gaussian wave function fit does a reasonably accurate job in the coalescence factor calculation (valid to a factor of 2 or so). What does turn out to be quantitatively important, as already noted above, is the consideration of the two different length scales associated with the $p-n$ and $\Lambda$-pn factors of the state.

In Sec. IV we give a rudimentary comparison to data. In Sec. IV A we recap results from [19] for D and ${ }^{3} \mathrm{He}$, adding a $p \mathrm{~Pb}$ data point to the $\mathrm{PbPb}$ and $p p$ measurements discussed there; we also correct a few typos in Ref. [19]. In Sec. IV B we compare the coalescence prediction for ${ }_{\Lambda}^{3} \mathrm{H}$ to $\mathrm{PbPb}$ data. We emphasize (as was done before us [22-24]; but here with a robust coalescence calculation) that ${ }_{\Lambda}^{3} \mathrm{H}$ data in small systems ( $p p, p \mathrm{~Pb}$, or low multiplicity $\mathrm{PbPb}$ ) has the potential to conclusively rule out (or support) coalescence as the dominant origin of clusters.

In Sec. $\mathrm{V}$ we discuss and summarize our results.

\section{THE COALESCENCE/FEMTOSCOPY FRAMEWORK}

The description of femtoscopic correlations between nucleons [25] and the coalescence model for nuclei [26,27] are two aspects of the same theoretical framework. The idea is that at kinetic freeze-out the HXS can be described by a multiparticle density matrix $\hat{\rho}_{\mathrm{HX}}$. Depending on the measured observable, this density matrix can be projected onto final states of different multiplicities. In what follows we present a concise derivation of coalescence and the coalescencecorrelations relation [19], aiming to collect different aspects of the problem under the same roof, so to speak. Many of the results were also derived elsewhere, notably in Refs. [16-19] and (albeit with model dependence) in Refs. [15,28-36].

\section{A. Two-particle correlations and the deuteron}

If the phase space density of nucleons around the time when they last scatter against other particles (mostly pions) in the HXS is not too high, then the subsequent propagation of pairs of nucleons emitted very near in phase space would be dominated by FSI, while additional interactions with particles other than the pair would be subdominant. With this sudden approximation, ${ }^{6}$ the Lorentz-invariant yield of nucleon pairs at total spin $s$ is given by $[16,18]$

$$
\begin{aligned}
\gamma_{1} \gamma_{2} \frac{d N_{2, s}}{d^{3} \mathbf{p}_{1} d^{3} \mathbf{p}_{2}}= & \frac{2 s+1}{(2 \pi)^{6}} \int d^{4} x_{1} \int d^{4} x_{2} \int d^{4} x_{1}^{\prime} \\
& \times \int d^{4} x_{2}^{\prime} \Psi_{s, p_{1}, p_{2}}^{*}\left(x_{1}^{\prime}, x_{2}^{\prime}\right) \Psi_{s, p_{1}, p_{2}}\left(x_{1}, x_{2}\right) \\
& \times \rho_{p_{1}, p_{2}}\left(x_{1}, x_{2} ; x_{1}^{\prime}, x_{2}^{\prime}\right)
\end{aligned}
$$

where $\Psi_{s, p_{1}, p_{2}}\left(x_{1}, x_{2}\right)$ is the continuum Bethe-Salpeter amplitude [39] describing the FSI of the pair. Similarly, the yield of deuterons at momentum $P$ is given by

$$
\begin{aligned}
\gamma \frac{d N_{\mathrm{d}}}{d^{3} \mathbf{P}}= & \frac{2 s_{d}+1}{(2 \pi)^{3}} \int d^{4} x_{1} \int d^{4} x_{2} \int d^{4} x_{1}^{\prime} \\
& \times \int d^{4} x_{2}^{\prime} \Psi_{d, P}^{*}\left(x_{1}^{\prime}, x_{2}^{\prime}\right) \Psi_{d, P}\left(x_{1}, x_{2}\right) \\
& \times \rho_{p_{1}, p_{2}}\left(x_{1}, x_{2} ; x_{1}^{\prime}, x_{2}^{\prime}\right),
\end{aligned}
$$

where $\Psi_{d, P}\left(x_{1}, x_{2}\right)$ is the bound state Bethe-Salpeter amplitude describing the deuteron. The role of the Bethe-Salpeter amplitudes $\Psi$ is to resum soft diagonal (ladder) FSI diagrams, factoring their effect out of an assumed underlying shortdistance amplitude forming the density matrix $\rho_{p_{1}, p_{2}}$.

Obviously, modeling the spectra Eqs. (1) and (2) requires proper modeling of the FSI, that are either calculable from first principles (in the case of Coulomb) or measurable (in the case of nuclear scattering amplitudes) and that we assume to be known. Modeling $\rho_{p_{1}, p_{2}}$ from first principles, however, is currently impossible. Our goal in this section, and in the rest of the paper, is to demonstrate that even without a priori knowledge of $\rho_{p_{1}, p_{2}}$, the mere fact that the same $\rho_{p_{1}, p_{2}}$ occurs in both of Eqs. (1) and (2) is enough to allow a model-independent, approximate prediction of the deuteron (and, with some added assumptions, other clusters) spectrum based on measurements of femtoscopic correlations [19].

Let us define $c_{1,2}=\left(p_{1,2} P\right) / P^{2}$, where the pair total momentum is $P=p_{1}+p_{2} \equiv 2 p$. With these, we define the center-of-mass coordinate $X=c_{1} x_{1}+c_{2} x_{2}$, the relative momentum $^{7} q=c_{2} p_{1}-c_{1} p_{2}$, and the relative coordinate $x=$ $x_{1}-x_{2}$. Both for two-particle correlations and for loosely bound nuclei, we are interested in nucleon pairs that are nonrelativistic in the pair rest frame (PRF), $\mathbf{q}^{2} \ll m^{2}$, and can neglect corrections of $O\left(\mathbf{q}^{2} / \mathrm{m}^{2}\right)$. In this case, the dependence of the amplitude $\Psi$ (be it $\Psi_{s, p_{1}, p_{2}}$ or $\Psi_{d, P}$ ) on the pair total momentum and center-of-mass coordinate can be approximately

\footnotetext{
${ }^{6}$ See $[1,26,37]$ for nonrelativistic formulations. See [38] for a discussion of corrections to the sudden approximation due to the residual charge of the HXS, as applied to low energy (80 MeV/nucleon) HIC

${ }^{7}$ Note $c_{1}+c_{2}=1, q P=0$ and $\left(p_{1}-p_{2}\right) P=\left(c_{1}-c_{2}\right) P^{2}=m_{1}^{2}-$ $m_{2}^{2}$.
} 
factored out from the dependence on the relative momentum and relative coordinate, ${ }^{8}$ via

$$
\Psi\left(x_{1}, x_{2}\right)=e^{-i P X} \phi(x) .
$$

Using Eq. (3) and changing to convenient coordinates, we can rewrite Eqs. (1) and (2) as

$$
\begin{aligned}
\gamma_{1} \gamma_{2} \frac{d N_{2, s}}{d^{3} \mathbf{p}_{1} d^{3} \mathbf{p}_{2}}= & \frac{2 s+1}{(2 \pi)^{6}} \int d^{4} r \\
& \times \int \frac{d^{4} k}{(2 \pi)^{4}} \tilde{\mathcal{D}}_{s, q}(k, r) \tilde{S}_{p_{1}, p_{2}}(k, r), \\
\gamma \frac{d N_{\mathrm{d}}}{d^{3} \mathbf{P}}= & \frac{2 s_{d}+1}{(2 \pi)^{3}} \int d^{4} r \\
& \times \int \frac{d^{4} k}{(2 \pi)^{4}} \tilde{\mathcal{D}}_{d}(k, r) \tilde{S}_{p_{1}, p_{2}}(k, r),
\end{aligned}
$$

where we define the relativistic internal Wigner density

$$
\tilde{\mathcal{D}}(k, r)=\int d^{4} \zeta e^{i k \zeta} \phi\left(r+\frac{\zeta}{2}\right) \phi^{*}\left(r-\frac{\zeta}{2}\right)
$$

(with $\tilde{\mathcal{D}}_{s, q}$ and $\tilde{\mathcal{D}}_{d}$ obtained from $\phi_{s, q}$ and $\phi_{d}$, respectively) and where

$$
\begin{aligned}
\tilde{S}_{p_{1}, p_{2}}(k, r)= & \int d^{4} x \int d^{4} l_{1} e^{-i l_{1}\left(c_{1} P+k\right)} \int d^{4} l_{2} e^{-i l_{2}\left(c_{2} P-k\right)} \\
& \times \rho_{p_{1}, p_{2}}\left(x+c_{2} r+\frac{l_{1}}{2}, x-c_{1} r+\frac{l_{2}}{2} ; x\right. \\
& \left.+c_{2} r-\frac{l_{1}}{2}, x-c_{1} r-\frac{l_{2}}{2}\right)
\end{aligned}
$$

The Wigner density we would obtain if we could turn off both FSI and quantum statistics (QS) is $\mathcal{D}_{s, q}^{0}(k, r)=$ $(2 \pi)^{4} \delta^{(4)}(k-q)$, independent of $r$. With this in mind, and with an eye to Eq. (4), we can define a hypothetical reference uncorrelated (and unpolarized) pair spectrum,

$$
\gamma_{1} \gamma_{2} \frac{d N_{2}^{0}}{d^{3} \mathbf{p}_{1} d^{3} \mathbf{p}_{2}}=\frac{\left(2 s_{N}+1\right)^{2}}{(2 \pi)^{6}} \int d^{4} r \tilde{S}_{p_{1}, p_{2}}^{0}(q, r),
$$

where $s_{N}=1 / 2$ is the nucleon spin. We stress that the reference pair spectrum of Eq. (8) is not a real physical quantity: obviously, we cannot really turn off FSI and QS. To highlight this fact we add the superscript ${ }^{0}$ on the object $\tilde{S}_{p_{1}, p_{2}}^{0}$, to distinguish it from $\tilde{S}_{p_{1}, p_{2}}$ entering Eqs. (4) and (5). ${ }^{9}$

We can try to mimic the reference pair spectrum experimentally by pairing particles from different events with

\footnotetext{
${ }^{8}$ In general, had we been interested in relativistic motion of the nucleons in the PRF, we would need to keep track of nontrivial dependence of the PRF amplitude on the total momentum, $\phi(x) \rightarrow$ $\phi_{P}(x)$.

${ }^{9}$ Even if we could turn off FSI and QS we would still not be guaranteed that the HXS provides $\tilde{S}_{p_{1}, p_{2}}^{0}=\tilde{S}_{p_{1}, p_{2}}$; for this identification to hold, density matrix factorization, introduced in Sec. II B, needs to be satisfied. But our definition of $\tilde{S}_{p_{1}, p_{2}}^{0}$ is merely an auxiliary: the results in the current section up to and including Eq. (18) do not depend on density matrix factorization and do not require $\tilde{S}_{p_{1}, p_{2}}^{0}=\tilde{S}_{p_{1}, p_{2}}$.
}

similar event characteristics. With this understanding, the pair correlation function is defined as

$$
C(p, q)=\frac{\sum_{s} \gamma_{1} \gamma_{2} \frac{d N_{2, s}}{d^{3} \mathbf{p}_{1} d^{3} \mathbf{p}_{2}}}{\gamma_{1} \gamma_{2} \frac{d N_{2}^{0}}{d^{3} \mathbf{p}_{1} d^{3} \mathbf{p}_{2}}}
$$

and the coalescence factor for deuteron formation is defined as

$$
\mathcal{B}_{2}(p)=\frac{P^{0} \frac{d N_{\mathrm{d}}}{d^{3} \mathbf{p}}}{p_{1}^{0} p_{2}^{0} \frac{d N_{2}^{0}}{d^{3} \mathbf{p}_{1} d^{3} \mathbf{p}_{2}}} \approx \frac{2}{m} \frac{\gamma \frac{d N_{\mathrm{d}}}{d^{3} \mathbf{P}}}{\gamma_{1} \gamma_{2} \frac{d N_{2}^{0}}{d^{3} \mathbf{p}_{1} d^{3} \mathbf{p}_{2}}},
$$

where we approximated $m_{D} \approx 2 m$.

At this point it is useful to make two approximations:

Smoothness approximation. The smoothness approximation was discussed widely in the literature $[11,12,40]$. The version of this approximation we take here amounts to replacing $S_{p_{1}, p_{2}}(k, r) \approx S_{p_{1}, p_{2}}(0, r)$ in Eqs. (4) and (5). The $k$ integral of the $\tilde{\mathcal{D}}$ functions can then be done, yielding $\int \frac{d^{4} k}{(2 \pi)^{4}} \tilde{\mathcal{D}}(k, r)=|\phi(r)|^{2}$. Similarly, we replace $S_{p_{1}, p_{2}}^{0}(q, r) \approx$ $S_{p_{1}, p_{2}}^{0}(0, r)$ in the reference pair spectrum Eq. (8). The accuracy of the smoothness approximation is probably sufficient for our purpose in $\mathrm{PbPb}$ collisions; in $p p$ collisions we think that a careful assessment is still warranted.

Equal-time approximation. In the PRF we have $P=$ $(M, \mathbf{0}), q=(0, \mathbf{q})$ and $x=(t, \mathbf{x})$. A key point, derived clearly in Refs. [16,18] (see especially Appendix A of [18] for a detailed discussion), is that the Bethe-Salpeter amplitude in the PRF nonrelativistic limit is approximately independent of the PRF time:

$$
\phi(x)=\phi(\mathbf{x})\left[1+O\left(\frac{t}{m \mathbf{x}^{2}}\right)\right] .
$$

At the level of the leading term in the equal-time approximation of Eq. (11), $\phi_{s, q}\left(\phi_{d}\right)$ is equal to the QM static scattering wave (bound state) solution of the Schrödinger equation [41].

In hadronic collisions we have $t \approx \mathbf{x} \approx$ few fm, implying a correction of order $0.2 \frac{1 \mathrm{fm}}{\mathrm{x}}$ to the equal-time approximation. Thus, we are certainly sacrificing some precision when we adopt it: the incurred theoretical error is probably about $10 \%$ for $\mathrm{PbPb}$ collisions, where $\mathbf{x} \approx 3 \mathrm{fm}$, but could be several tens of percent in $p p$ collisions where $\mathbf{x} \approx 1 \mathrm{fm}$. Nevertheless, the equal-time approximation would allow us significant utility with relatively simple notation. We think that it probably allows for sufficient accuracy to establish (or exclude) coalescence as the main origin of clusters in hadronic collisions. Note that the free solution in the absence of FSI is the plane wave $\phi(x)=e^{-i q x}$ [(anti)symmetrized by QS] and the equaltime limit of Eq. (11) is exact in that case, because in the PRF $q x=-\mathbf{q x}$.

Adopting the smoothness and equal-time approximations, we are led to the definition ${ }^{10}$ of the normalized two-particle source $\mathcal{S}_{2}$, a function of the PRF spatial coordinate $\mathbf{r}$, as an

\footnotetext{
${ }^{10}$ It is common practice (e.g., $\left.[20,42,43]\right)$ to drop the explicit mention of $p$ in $\mathcal{S}_{2}(\mathbf{r})$; this, despite the fact that $\mathcal{S}_{2}(\mathbf{r})$ does depend on $p$. As long as we keep this fact in mind, this practice brings no harm.
} 
integral in PRF time $t=r^{0}$ :

$$
\mathcal{S}_{2}(\mathbf{r})=\frac{\int d r^{0} \tilde{S}_{p_{1}, p_{2}}(0, r)}{\int d^{4} r \tilde{S}_{p_{1}, p_{2}}^{0}(0, r)} .
$$

The pair correlation function is then given from Eq. (9) as

$$
C(p, q)=\sum_{s} w_{s} C_{s}(p, q)
$$

with the spin weights $w_{s}=(2 s+1) /\left(2 s_{N}+1\right)^{2}$ and

$$
C_{s}(p, q) \approx \int d^{3} \mathbf{r}\left|\phi_{s, q}(\mathbf{r})\right|^{2} \mathcal{S}_{2}(\mathbf{r}) .
$$

Similarly, the deuteron coalescence factor is given from Eq. (10) as

$$
\mathcal{B}_{2}(p) \approx \frac{2\left(2 s_{d}+1\right)}{m\left(2 s_{N}+1\right)^{2}}(2 \pi)^{3} \int d^{3} \mathbf{r}\left|\phi_{d}(\mathbf{r})\right|^{2} \mathcal{S}_{2}(\mathbf{r}) .
$$

Two general comments are in order. First, the aim of femtoscopy analyses like, e.g., Refs. [20,42,43] (for reviews, see $[11,12])$ is to measure the source $\mathcal{S}_{2}(\mathbf{r})$ by solving the Schrödinger equation for the wave functions $\phi_{s, q}(\mathbf{r})$ $[18,25,44]$ and comparing the observed two-particle spectrum with Eqs. (13) and (14). In this exercise, obtaining agreement with the experimental data appears to require the use of the correct set of FSI potentials. We would thus like to emphasize that the success of the source reconstruction analyses lends some credence to the basic framework leading to Eqs. (13) and (14). As we have just seen, this is the same framework that stands behind the coalescence model for nuclei, the only difference being that femtoscopy deals with continuum scattering state solutions of FSI and coalescence deals with discrete bound state solutions of FSI. Thus the mere existence of successful femtoscopy analyses lends some credence to coalescence as the origin of (at least some of the) nuclei observed in hadronic collisions. In the future, it might even be possible to observe a trace of the bound state formation directly on the correlation function of, for instance, $d-\Lambda$ or $d-p$ [45].

Second, with $\mathcal{S}_{2}(\mathbf{r})$ measured, Eq. (15) predicts the deuteron yield model independently with no free parameters. As we show in Sec. IV, this prediction is consistent with experimental data to within a factor of 2 or so across systems ranging from $\mathrm{PbPb}$ to $p \mathrm{~Pb}$ and $p p$ at different multiplicities $[19,36]$. This is strong evidence that coalescence contributes to the production of deuterons in hadronic collisions at the $O(1)$ level, at least.

A caveat to keep in mind is that, although Eq. (14) is commonly used in the literature, we do not know a modelindependent way of checking the quantitative corrections due to the smoothness and equal-time approximations, which we have done to reduce Eq. (4) into Eq. (14); or the sudden approximation itself, allowing us to write Eq. (4) in the first place. A systematic study of these uncertainties is warranted if one wishes to narrow down the theory uncertainty associated with Eq. (15).

As a technical aside, it is convenient to carry out some of the analysis in momentum space. To this end it is useful to introduce the momentum space function $\mathcal{C}_{2}$, which is just the
Fourier transform of $\mathcal{S}_{2}(\mathbf{r})$ :

$$
\mathcal{C}_{2}(p, \mathbf{k})=\int d^{3} \mathbf{r} e^{i \mathbf{k r}} \mathcal{S}_{2}(\mathbf{r}) .
$$

(We will usually keep the explicit appearance of $p$ in $\mathcal{C}_{2}$.) Defining the momentum space deuteron form factor $\mathcal{F}_{d}$

$$
\left|\phi_{d}(\mathbf{r})\right|^{2}=\int \frac{d^{3} \mathbf{k}}{(2 \pi)^{3}} e^{i \mathbf{k r}} \mathcal{F}_{d}(\mathbf{k}),
$$

we can rewrite Eq. (15) as

$$
\mathcal{B}_{2}(p) \approx \frac{2\left(2 s_{d}+1\right)}{m\left(2 s_{N}+1\right)^{2}} \int d^{3} \mathbf{k} \mathcal{F}_{d}(\mathbf{k}) \mathcal{C}_{2}(p, \mathbf{k}) .
$$

This is the version of the coalescence-correlation relation derived in Ref. [19].

\section{B. Connecting two-particle states with one- and three-particle states: Density matrix factorization}

While two-particle femtoscopic correlations are directly and model-independently connected to deuteron coalescence, the connection to single-particle spectra and to the coalescence of three-body states requires further assumptions. The main assumption we need is the factorization of the multiparticle density matrix into the product of single-particle density matrices (see, e.g., [25,27]),

$$
\rho_{p_{1}, p_{2}}\left(x_{1}, x_{2} ; x_{1}^{\prime}, x_{2}^{\prime}\right) \approx \rho_{p_{1}}\left(x_{1} ; x_{1}^{\prime}\right) \rho_{p_{2}}\left(x_{2} ; x_{2}^{\prime}\right) .
$$

Factorization cannot be exact. For example, in low multiplicity events total momentum conservation inevitably leads to the breakdown of Eq. (19), seen observationally as "nonfemtoscopic correlations" at large q. Nevertheless, keeping these caveats in mind we will adopt the factorization approximation in what follows. Later on we investigate one simple way to parametrize related corrections.

\section{Uncorrelated pair spectrum as a product of one-particle spectra}

Considering nucleons (protons and neutrons) with $m_{1} \approx$ $m_{2} \approx m$, nonrelativistic in the PRF, we have $q \approx p_{1}-p_{2}$ and the factors $c_{1} \approx c_{2}=\frac{1}{2}+O\left(\mathbf{q}^{2} / \mathrm{m}^{2}\right)$. Inserting this into Eq. (7) and using Eq. (19) leads to

$$
\tilde{S}_{p_{1}, p_{2}}(q, r) \approx \int d^{4} x \tilde{S}_{p_{1}}\left(x+\frac{r}{2}\right) \tilde{S}_{p_{2}}\left(x-\frac{r}{2}\right),
$$

with the one-particle emission function

$$
\tilde{S}_{p}(x)=\int d^{4} l e^{-i l p} \rho_{p}\left(x+\frac{l}{2} ; x-\frac{l}{2}\right) .
$$

This $\tilde{S}_{p}(x)$ coincides (up to constant factors in the definition) with the particle source of [25] and with the emission function or phase space density of $[11,12,15,46,47]$. The reference pair spectrum factorizes into the product of single-particle spectra,

$$
\begin{aligned}
\gamma_{1} \gamma_{2} \frac{d N_{2}^{0}}{d^{3} \mathbf{p}_{1} d^{3} \mathbf{p}_{2}} & \approx\left[\gamma_{1} \frac{d N}{d^{3} \mathbf{p}_{1}}\right]\left[\gamma_{2} \frac{d N}{d^{3} \mathbf{p}_{2}}\right], \\
\gamma \frac{d N}{d^{3} \mathbf{p}} & =\frac{\left(2 s_{N}+1\right)}{(2 \pi)^{3}} \int d^{4} x \tilde{S}_{p}(x) .
\end{aligned}
$$


Finally, the two-particle source $\mathcal{S}_{2}$ is constructed from single-particle emission functions as

$$
\mathcal{S}_{2}(\mathbf{r})=\frac{\int d r^{0} \int d^{4} x \tilde{S}_{p}\left(x+\frac{r}{2}\right) \tilde{S}_{p}\left(x-\frac{r}{2}\right)}{\left[\int d^{4} x \tilde{S}_{p}(x)\right]^{2}} .
$$

It can be more convenient to calculate $\mathcal{C}_{2}(p, \mathbf{q})$, by inserting Eq. (24) into Eq. (16), giving the prescription

$$
\mathcal{C}_{2}(p, \mathbf{q})=\frac{\left|\int d^{4} x e^{i q x} \tilde{S}_{p}(x)\right|^{2}}{\left[\int d^{4} x \tilde{S}_{p}(x)\right]^{2}}
$$

In evaluating Eq. (25), recall that we require $q=(0, \mathbf{q})$ as specified in the PRF.

As a slight detour, consider the proton pair correlation with FSI turned off but quantum statistics still on, in the spinasymmetric or spin-symmetric state where $\phi_{s, q}(\mathbf{r})=\frac{1}{\sqrt{2}}\left(e^{i \mathbf{q r}} \pm e^{-i \mathbf{q r}}\right)$, respectively. Using Eq. (24) and noting that $q^{0}=0$ in the PRF, the pair correlation of Eq. (14) would be

$$
C_{s}(p, q) \approx \int d^{3} \mathbf{r}\left|\phi_{s, q}(\mathbf{r})\right|^{2} \mathcal{S}_{2}(\mathbf{r})=1 \pm \frac{\left|\int d^{4} x e^{2 i q x} \tilde{S}_{p}(x)\right|^{2}}{\left[\int d^{4} x \tilde{S}_{p}(x)\right]^{2}}=1 \pm \mathcal{C}_{2}(p, 2 \mathbf{q}) \quad \text { (limit of no FSI) }
$$

consistent with the usual expression in the literature [11,12,47,48] (note that $q$ as defined in, e.g., [48] is equal to $2 q$ in our notation).

\section{Hypertriton and ${ }^{3} \mathrm{He}$}

The starting point in the coalescence calculation for the hypertriton ${ }_{\Lambda}^{3} \mathrm{H}(p n \Lambda)$ is similar to Eq. (2) for the deuteron:

$$
\begin{aligned}
\gamma \frac{d N_{\Lambda} \mathrm{H}}{d^{3} \mathbf{P}}= & \frac{2 s_{\Lambda}^{3} \mathrm{H}+1}{(2 \pi)^{3}} \int d^{4} x_{p} \int d^{4} x_{n} \int d^{4} x_{\Lambda} \int d^{4} x_{p}^{\prime} \int d^{4} x_{n}^{\prime} \int d^{4} x_{\Lambda}^{\prime} \\
& \times \Psi_{{ }_{\Lambda} \mathrm{H}, P}^{*}\left(x_{p}^{\prime}, x_{n}^{\prime}, x_{\Lambda}^{\prime}\right) \Psi_{\Lambda}{ }_{{ }^{3} \mathrm{H}, P}\left(x_{p}, x_{n}, x_{\Lambda}\right) \rho_{p_{p}, p_{n}, p_{\Lambda}}\left(x_{p}, x_{n}, x_{\Lambda} ; x_{p}^{\prime}, x_{n}^{\prime}, x_{\Lambda}^{\prime}\right),
\end{aligned}
$$

where $\Psi_{{ }^{3} \mathrm{H}, P}\left(x_{p}, x_{n}, x_{\Lambda}\right)$ is the bound state Bethe-Salpeter amplitude describing the ${ }_{\Lambda}^{3} \mathrm{H}$. The total momentum is $P=p_{p}+p_{n}+$ $p_{\Lambda} \equiv 3 p$. We also define $P_{p n}=p_{p}+p_{n}, c_{I}=\left(p_{I} P\right) / P^{2}$ with $I=p, n, \Lambda$, and $\tilde{c}_{J}=\left(p_{J} P_{p n}\right) / P_{p n}^{2}$ with $J=p, n$. The center-ofmass coordinate is then $X=c_{n} x_{n}+c_{p} x_{p}+c_{\Lambda} x_{\Lambda}$, and useful relative coordinates are $r_{p n}=x_{p}-x_{n}$ and $r_{\Lambda}=x_{\Lambda}-\tilde{c}_{p} x_{p}-\tilde{c}_{n} x_{n}$. With these definitions the Bethe-Salpeter amplitude factorizes into $\Psi_{{ }^{3} \mathrm{H}, P}=e^{-i P X} \phi_{\Lambda_{3} \mathrm{H}}\left(r_{p n}, r_{\Lambda}\right)$.

The calculation for ${ }^{3} \mathrm{He}(p p n)$ is similar, with the replacements $r_{p n} \rightarrow r_{p p}$ and $r_{\Lambda} \rightarrow r_{n}$, etc.

Following the same steps as in Sec. II A and adding to that the smoothness approximation, the equal-time approximation, as well as density matrix factorization à la Eq. (19) extended to three particles, we are led,eventually, to the normalized three-particle source expressed as integrals of single-particle emission functions,

$$
\begin{aligned}
\mathcal{S}_{3 \Lambda}\left(\mathbf{r}_{p n}, \mathbf{r}_{\Lambda}\right) & =\frac{\int d r_{p n}^{0} \int d r_{\Lambda}^{0} \int d^{4} x \tilde{S}_{p}\left(x+\frac{r_{p n}}{2}-\frac{r_{\Lambda}}{3}\right) \tilde{S}_{p}\left(x-\frac{r_{p n}}{2}-\frac{r_{\Lambda}}{3}\right) \tilde{S}_{p}^{(\Lambda)}\left(x+\frac{2 r_{\Lambda}}{3}\right)}{\left[\int d^{4} x \tilde{S}_{p}(x)\right]^{2} \int d^{4} x \tilde{S}_{p}^{(\Lambda)}(x)}, \\
\mathcal{S}_{3}\left(\mathbf{r}_{p p}, \mathbf{r}_{n}\right) & =\frac{\int d r_{p p}^{0} \int d r_{n}^{0} \int d^{4} x \tilde{S}_{p}\left(x+\frac{r_{p p}}{2}-\frac{r_{n}}{3}\right) \tilde{S}_{p}\left(x-\frac{r_{p p}}{2}-\frac{r_{n}}{3}\right) \tilde{S}_{p}\left(x+\frac{2 r_{n}}{3}\right)}{\left[\int d^{4} x \tilde{S}_{p}(x)\right]^{3}} .
\end{aligned}
$$

The coalescence factors are then found as

$$
\begin{aligned}
\mathcal{B}_{3 \Lambda}(p) & \approx \frac{3}{m^{2}} \frac{2 s_{\Lambda}^{3} \mathrm{H}+1}{\left(2 s_{N}+1\right)^{3}}(2 \pi)^{6} \int d^{3} \mathbf{r}_{p n} \int d^{3} \mathbf{r}_{\Lambda}\left|\phi_{\Lambda} \mathrm{H}\left(\mathbf{r}_{p n}, \mathbf{r}_{\Lambda}\right)\right|^{2} \mathcal{S}_{3 \Lambda}\left(\mathbf{r}_{p n}, \mathbf{r}_{\Lambda}\right), \\
\mathcal{B}_{3}(p) & \approx \frac{3}{m^{2}} \frac{2 s_{\mathrm{He}}+1}{\left(2 s_{N}+1\right)^{3}}(2 \pi)^{6} \int d^{3} \mathbf{r}_{p p} \int d^{3} \mathbf{r}_{n}\left|\phi_{{ }^{3} \mathrm{He}}\left(\mathbf{r}_{p p}, \mathbf{r}_{n,}\right)\right|^{2} \mathcal{S}_{3}\left(\mathbf{r}_{p p}, \mathbf{r}_{n}\right) .
\end{aligned}
$$

We highlight that in the $\mathcal{S}_{3 \Lambda}$ calculation one emission function corresponds to the emission of a $\Lambda$, rather than a nucleon. For simplicity we approximated $m_{\Lambda} \approx m$ in the prefactor, at the cost of an error of about $10 \%$.

Again, we can define the Fourier transform

$$
\mathcal{C}_{3 \Lambda}\left(p, \mathbf{q}_{1}, \mathbf{q}_{2}\right)=\int d^{3} \mathbf{r}_{1} \int d^{3} \mathbf{r}_{2} e^{i \mathbf{q}_{1} \mathbf{r}_{1}+i \mathbf{q}_{2} \mathbf{r}_{2}} \mathcal{S}_{3 \Lambda}\left(\mathbf{r}_{1}, \mathbf{r}_{2}\right)
$$

and the momentum space form factor $\mathcal{F}_{{ }^{3}} \mathrm{H}$,

$$
\left|\phi_{3_{\Lambda} \mathrm{H}}\left(\mathbf{r}_{p n}, \mathbf{r}_{\Lambda}\right)\right|^{2}=\int \frac{d^{3} \mathbf{k}_{p n}}{(2 \pi)^{3}} e^{i \mathbf{k}_{d} \mathbf{r}_{p n}} \int \frac{d^{3} \mathbf{k}_{\Lambda}}{(2 \pi)^{3}} e^{i \mathbf{k}_{\Lambda} \mathbf{r}_{\Lambda}} \mathcal{F}_{{ }_{\Lambda}} \mathrm{H}\left(\mathbf{k}_{p n}, \mathbf{k}_{\Lambda}\right) .
$$


In terms of these (and their equivalents for ${ }^{3} \mathrm{He}$ ) the coalescence factors read

$$
\begin{aligned}
\mathcal{B}_{3 \Lambda}(p) & \approx \frac{3}{m^{2}} \frac{2 s_{\Lambda}^{3} \mathrm{H}}{\left(2 s_{N}+1\right)^{3}} \int d^{3} \mathbf{k}_{p n} \int d^{3} \mathbf{k}_{\Lambda} \mathcal{F}_{{ }_{\Lambda}} \mathrm{H} \\
\mathcal{B}_{3}(p) & \left.\approx \frac{3}{m^{2}} \frac{\left.2 \mathbf{k}_{p n}, \mathbf{k}_{\Lambda}\right) \mathcal{C}_{3 \Lambda}(p, 1}{\left(2 s_{N}+1\right)^{3}} \int d^{3} \mathbf{k}_{p n}, \mathbf{k}_{\Lambda}\right)
\end{aligned}
$$

The coalescence of hypertriton and ${ }^{3} \mathrm{He}$ can be connected to femtoscopy analyses, to the extent that the three-particle normalized sources $\mathcal{S}_{3 \Lambda}$ and $\mathcal{S}_{3}$ can be accessed by correlation measurements. This connection would be crucial for the attempt to use ${ }_{\Lambda}^{3} \mathrm{H}$, due to its large nucleus size, as a test of the coalescence framework. However, the connection is not as direct as it is for deuteron formation. We discuss this connection further in the next section.

\section{COALESCENCE FROM CORRELATION FUNCTIONS}

\section{A. Femtoscopy source parametrization}

We now want to make practical contact with observational information on the HXS source size, available from femtoscopy studies. Experimental analyses commonly fit the measured two-particle source using a Gaussian approximation $[20,42,43]$, the simplest version of which is the isotropic (or 1D) Gaussian [16]

$$
\mathcal{S}_{2}^{1 D}(\mathbf{r})=\frac{1}{\left(4 \pi R_{\text {inv }}^{2}\right)^{\frac{3}{2}}} e^{-\frac{\mathbf{r}^{2}}{4 R_{\mathrm{inv}}^{2}}} .
$$

The radius parameter $R_{\text {inv }}$ depends on $p[20,42,43]$.

From the theoretical perspective $[11,12,40] \mathrm{Eq}$. (36) could arise, for example, if the emission function $\tilde{S}_{p}(x)$ receives most of its support near some configuration space location $\mathbf{R}_{s}$ and is also sharply peaked around kinetic freeze-out time $t_{f}$, as measured in the emitted particle rest frame, allowing the approximation

$$
\tilde{S}_{p}(x) \propto e^{-\frac{(\mathbf{r}-\mathbf{R})^{2}}{2 R_{\text {inv }}^{2}}} \delta\left(t-t_{f}\right) .
$$

Inserting Eq. (37) into Eq. (24) immediately yields Eq. (36). While more difficult to test experimentally [49], Eq. (37) also predicts $\mathcal{S}_{3}$ of Eqs. (28) and (29),

$$
\begin{aligned}
\mathcal{S}_{3}^{1 D}\left(\mathbf{r}_{1}, \mathbf{r}_{2}\right) & =\frac{\int d^{3} \mathbf{x} e^{-\frac{\left(\mathbf{x}+\frac{\mathbf{r}_{1}}{2}-\frac{\mathbf{r}_{2}}{3}-\mathbf{R}_{s}\right)^{2}+\left(\mathbf{x}-\frac{\mathbf{r}_{1}}{2}-\frac{\mathbf{r}_{2}}{3}-\mathbf{R}_{s}\right)^{2}+\left(\mathbf{x}+\frac{2 \mathbf{r}_{2}}{3}-\mathbf{R}_{s}\right)^{2}}{2 R_{\mathrm{inv}}^{2}}}}{\left[\int d^{3} \mathbf{x} e^{-\frac{\left(\mathbf{x}-\mathbf{R}_{s}\right)^{2}}{2 R_{\mathrm{inv}}^{2}}}\right]^{3}} \\
& =\frac{1}{\left(12 \pi^{2} R_{\mathrm{inv}}^{4}\right)^{\frac{3}{2}}} e^{-\frac{\mathbf{r}_{1}^{2}+\frac{4}{3} \mathbf{r}_{2}^{2}}{4 R_{\mathrm{inv}}^{2}}}
\end{aligned}
$$

The momentum space versions of the isotropic Gaussian source model are

$$
\begin{aligned}
\mathcal{C}_{2}^{1 D}(p, \mathbf{q}) & =e^{-\mathbf{q}^{2} R_{\mathrm{inv}}^{2}} \\
\mathcal{C}_{3}^{1 D}\left(p, \mathbf{q}_{1}, \mathbf{q}_{2}\right) & =e^{-R_{\mathrm{inv}}^{2}\left(\mathbf{q}_{1}^{2}+\frac{3}{4} \mathbf{q}_{2}^{2}\right)} .
\end{aligned}
$$

Note that here we have considered the correlation of particles (e.g., protons) with the same underlying emission function $\tilde{S}_{p}(x)$. Soon, however, we will use Eq. (40) to analyze ${ }_{\Lambda}^{3} \mathrm{H}$ production which involves both $\tilde{S}_{p}(x)$ and $\tilde{S}_{p}^{(\Lambda)}(x)$, and there is no guarantee that these emission functions involve the same values of $\mathbf{R}_{s}$ and $R_{\text {inv }}$ in Eq. (37). Experimental results in $p p$ collisions [20] suggest that $R_{\text {inv }}$ as extracted from $p \Lambda$ correlations actually differs from $R_{\text {inv }}$ extracted from $p p$ correlations by $20 \%$ or so. ${ }^{11}$ It must then be understood that, in using our formalism to compute ${ }_{\Lambda}^{3} \mathrm{H}$, any effective value we use for $R_{\text {inv }}$ in Eqs. (38) or (40) cannot be more accurately determined than the aforementioned $20 \%$, without introducing model-dependent assumptions concerning the behavior of the emission functions. We will comment on this point again in Sec. IV B when we discuss the comparison between the ${ }_{\Lambda}^{3} \mathrm{H}$ coalescence prediction and experimental data.

The isotropic Gaussian source model is quite unrealistic. Even if the HXS "fireball" was somehow isotropic in the laboratory frame (generally it is not; e.g., [11,48]), it would be seen as anisotropic in the PRF due to Lorentz boost along the direction of $\mathbf{p}$. In addition, the beam line, of course, is a special direction in the initial state forming the HXS. As a simple generalization that can capture some of these effects (and others), one can consider an anisotropic (3D) Gaussian source with a free normalization: ${ }^{12}$

$$
\begin{aligned}
\mathcal{C}_{2}^{3 D}(p, \mathbf{q}) & =\mathcal{N}_{2} e^{-q_{l}^{2} R_{l}^{2}-q_{o}^{2} R_{o}^{2}-q_{s}^{2} R_{s}^{2}} \\
\mathcal{C}_{3}^{3 D}\left(p, \mathbf{q}_{1}, \mathbf{q}_{2}\right) & =\mathcal{N}_{3} e^{-R_{l}^{2}\left(q_{1 l}^{2}+\frac{3}{4} q_{2 l}^{2}\right)-R_{o}^{2}\left(q_{1 o}^{2}+\frac{3}{4} q_{2 o}^{2}\right)-R_{s}^{2}\left(q_{1 s}^{2}+\frac{3}{4} q_{2 s}^{2}\right)} .
\end{aligned}
$$

Here, we split the 3-vector $\mathbf{q}$ into its three components: $q_{o}$ ("out") along the direction of the mean transverse momentum $\mathbf{p}_{t} ; q_{l}$ ("longitudinal") along the beam axis; and $q_{s}$ ("side") along the third orthogonal direction $\mathbf{p}_{t} \times \hat{z}$.

Like the femtoscopic radii $R_{o, s, l}$, the normalization factors $\mathcal{N}_{2,3}$ (closely related to the "intercept" or chaoticity parameter [17,50]; see Appendix A for details) should best be measured directly from the data.

In Appendix A we briefly review how weak and strong resonance decays can distort the Gaussian shape of the source. In Appendix B we give numerical examples of $\mathcal{C}_{2}$ calculated in the phenomenological blast wave model, illustrating how anisotropic flow and Lorentz contraction at $p_{t}>0$ give rise to a true $3 \mathrm{D}$ source. We find that while the 1D Eq. (39)

\footnotetext{
${ }^{11}$ This is attributed in Ref. [20] to the different contributions of strong resonance decays to the $\mathrm{p}$ and $\Lambda$ spectra. From the point of view of our discussion, however, the cause of the difference in $R_{\text {inv }}$ is not essential.

${ }^{12}$ Adding off-diagonal components like $q_{o} q_{l} R_{o l}^{2}$, for example, into our analysis would be straightforward.
} 
can fail quite badly, the 3D Eq. (41) as a phenomenological parametrization is flexible enough to capture the true physical source to good accuracy.

Given the source parametrization of Eqs. (41) and (42), the final ingredients we need to evaluate Eqs. (18), (34), and (35) for the coalescence factors are the nucleus wave functions, encoded by the form factors $\mathcal{F}_{d,{ }^{3} \mathrm{He},{ }_{\Lambda}^{3} \mathrm{H}}$. We turn to that next.

\section{B. Nucleus wave functions: Gaussian wave function approximation}

The results are particularly tractable if we make the simplifying approximation of Gaussian wave functions. This is useful for analytic insight and is also reasonable if one wants to test coalescence at the $O(1)$ level, so we report the results in this section. In the next section we consider more accurate parametrizations of the wave functions.

For D we consider

$$
\phi_{d}(\mathbf{r})=\left(\frac{1}{\pi b_{d}^{2}}\right)^{\frac{3}{4}} e^{-\frac{\mathbf{r}^{2}}{2 b_{d}^{2}}},
$$

with momentum space form factor

$$
\mathcal{F}_{d}(\mathbf{k})=e^{-\frac{b_{d}^{2} \mathbf{k}^{2}}{4}} .
$$

vskip-4pt

For ${ }^{3} \mathrm{He}$ we consider a Gaussian that is isotropic in the normalized Jacobi coordinates, related to our natural kinematic coordinates $^{13}$ via $\eta_{p p}=\frac{1}{\sqrt{2}} \mathbf{r}_{p p}, \eta_{n}=\sqrt{\frac{2}{3}} \mathbf{r}_{n}$ :

$$
\phi_{{ }^{\mathrm{He}}}\left(\mathbf{r}_{p p}, \mathbf{r}_{n}\right)=\left(\frac{1}{3 \pi^{2} b_{3_{\mathrm{He}}^{4}}^{4}}\right)^{\frac{3}{4}} e^{-\frac{\mathbf{r}_{p p}^{2}+\frac{4}{3} \mathrm{r}_{n}^{2}}{4 b^{2} \mathrm{He}}} .
$$

With this $\phi_{3 \mathrm{He}}$ the momentum space form factor is

$\mathcal{F}^{{ }^{3} \mathrm{He}}\left(\mathbf{k}_{p p}, \mathbf{k}_{n}\right)=\int d^{3} \mathbf{r}_{p p} e^{-i \mathbf{k}_{\mathrm{pp}} \mathbf{r}_{p p}} \int d^{3} \mathbf{r}_{n} e^{-i \mathbf{k}_{\mathbf{n}} \mathbf{r}_{\mathbf{n}}}\left|\phi \mathrm{He}\left(\mathbf{r}_{p p}, \mathbf{r}_{n}\right)\right|^{2}$

$$
\begin{aligned}
\mathcal{B}_{2} & \approx \frac{12 \pi^{\frac{3}{2}} \mathcal{N}_{2}}{m\left(b_{d}^{2}+4 R_{l}^{2}\right)^{\frac{1}{2}}\left(b_{d}^{2}+4 R_{o}^{2}\right)^{\frac{1}{2}}\left(b_{d}^{2}+4 R_{s}^{2}\right)^{\frac{1}{2}}}, \\
\mathcal{B}_{3} & \approx \frac{16 \pi^{3} \mathcal{N}_{3}}{\sqrt{3} m^{2}\left(b_{3_{\mathrm{He}}^{2}}^{2}+2 R_{l}^{2}\right)\left(b_{{ }^{\mathrm{He}}}^{2}+2 R_{o}^{2}\right)\left(b_{{ }^{\mathrm{He}}}^{2}+2 R_{s}^{2}\right)}, \\
\mathcal{B}_{3 \Lambda} & \approx \frac{16 \pi^{3} \lambda_{3 \Lambda}}{\sqrt{3} m^{2}\left(b_{p n}^{2}+2 R_{l}^{2}\right)^{\frac{1}{2}}\left(b_{\Lambda}^{2}+2 R_{l}^{2}\right)^{\frac{1}{2}}\left(b_{p n}^{2}+2 R_{o}^{2}\right)^{\frac{1}{2}}\left(b_{\Lambda}^{2}+2 R_{o}^{2}\right)^{\frac{1}{2}}\left(b_{p n}^{2}+2 R_{s}^{2}\right)^{\frac{1}{2}}\left(b_{\Lambda}^{2}+2 R_{s}^{2}\right)^{\frac{1}{2}}} .
\end{aligned}
$$

We stress again that the analytic results obtained with Gaussian wave functions are brought here as means for an easy, rough assessment of the coalescence factor. More accurate calculations should use more accurate wave functions, especially for the $\mathrm{D}$ and ${ }_{\Lambda}^{3} \mathrm{H}$. We consider this refinement next.

\footnotetext{
${ }^{13}$ One can verify that $\int d^{3} \mathbf{r}_{p p} \int d^{3} \mathbf{r}_{n}\left|\phi_{3^{3} \mathrm{He}}\left(\mathbf{r}_{p p}, \mathbf{r}_{n}\right)\right|^{2}=$ (3) $)^{\frac{3}{2}} \int d^{3} \eta_{p p} \int d^{3} \eta_{n}\left(\frac{1}{3 \pi^{2} b_{3}^{4}}\right)^{\frac{3}{2}} e^{-\frac{\eta_{p p}^{2}+\eta_{n}^{2}}{b^{2}}}=1$.
}

$$
=e^{-\frac{b_{3 \mathrm{He}}^{2}}{2}\left(\mathbf{k}_{p p}^{2}+\frac{3}{4} \mathbf{k}_{n}^{2}\right)} .
$$

For ${ }_{\Lambda}^{3} \mathrm{H}$, as a first approximation we consider a product of Gaussians,

$$
\phi_{\Lambda} \mathrm{H}\left(\mathbf{r}_{p n}, \mathbf{r}_{\Lambda}\right) \approx\left(\frac{1}{3 \pi^{2} b_{p n}^{2} b_{\Lambda}^{2}}\right)^{\frac{3}{4}} e^{-\frac{\mathbf{r}_{p n}^{2}}{4 b_{p n}^{2}}-\frac{\mathbf{r}_{\Lambda}^{2}}{3 b_{\Lambda}^{2}}}
$$

The momentum space form factor is then

$$
\mathcal{F}_{\Lambda} \mathrm{H}\left(\mathbf{k}_{p n}, \mathbf{k}_{\Lambda}\right) \approx e^{-\frac{1}{2}\left(b_{p n}^{2} \mathbf{k}_{p n}^{2}+\frac{3}{4} b_{\Lambda}^{2} \mathbf{k}_{\Lambda}^{2}\right)} .
$$

We need to match the $b$ parameters to nuclear data. For D we take $b_{d}=3.5 \mathrm{fm}$, corresponding to the RMS charge radius $r_{\mathrm{rms}}=\sqrt{\frac{3}{8}} b_{d}=2.13 \mathrm{fm}$ [51]. For an isotropic three-body Gaussian wave function, the parameter $b$ as we defined it is directly the RMS charge radius. For ${ }^{3} \mathrm{He}$ this is $b_{3} \mathrm{He} \approx 1.97 \mathrm{fm}$ [51]. For ${ }_{\Lambda}^{3} \mathrm{H}$, Hildenbrand and Hammer [21] reported ${ }^{14}$ $\sqrt{\left\langle\mathbf{r}_{p n}^{2}\right\rangle}=\sqrt{3} b_{p n} \approx 3 \mathrm{fm}$ and $\sqrt{\left\langle\mathbf{r}_{\Lambda}^{2}\right\rangle}=\frac{3}{2} b_{\Lambda} \approx 10.8_{-1.5}^{+3} \mathrm{fm}$. It is important to note, however, that Ref. [21] reported the full numerical momentum space form factors and the $\sqrt{\left\langle\mathbf{r}^{2}\right\rangle}$ parameters they quote refer only to the small- $\mathbf{k}^{2}$ expansion of these form factors. A more accurate treatment of the wave function is obtained by using the full form factors, which we do in Sec. IIIC. Based on that analysis, for the Gaussian approximation we set $b_{p n}=1.52 \mathrm{fm}$ (as opposed to $b_{p n}=1.73 \mathrm{fm}$ that would be read from the low-k $\mathbf{k}^{2}$ fit) and $b_{\Lambda}=7.2_{-1}^{+2} \mathrm{fm}$.

With the Gaussian nuclear wave functions described in this section, assuming the femtoscopic source parametrization of Eqs. (41) and (42) and recalling the spins $s_{N}=s_{3} \mathrm{He}=s_{\lambda} \mathrm{H}=$ $\frac{1}{2}, s_{d}=1$, the coalescence factors evaluated from Eqs. (18), (34), and (35) are given by ${ }^{15}$ 


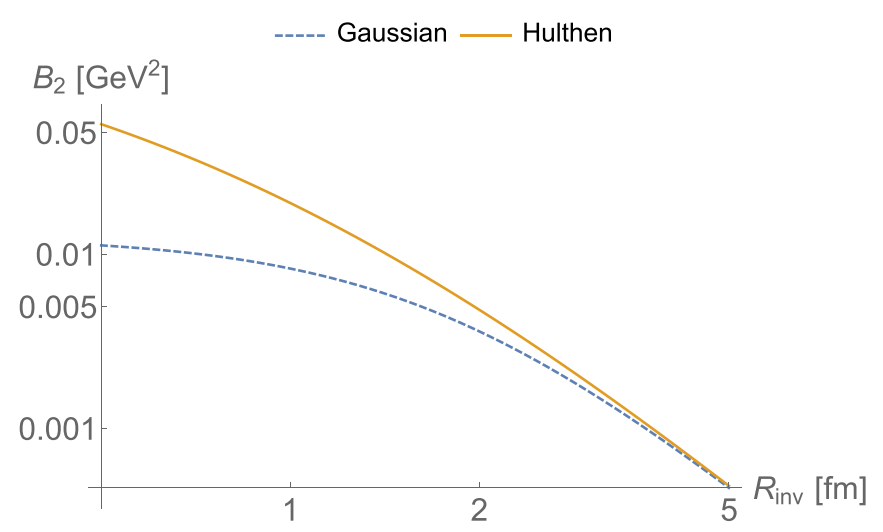

FIG. 1. Deuteron coalescence factor $\mathcal{B}_{2}$, calculated in the isotropic (1D) Gaussian source model, showing the difference between the Gaussian and Hulthen wave function parametrizations. We $\operatorname{set} \mathcal{N}_{2}=1$.

\section{Nucleus wave functions: More accurate parametrization}

Here we consider more accurate parametrizations for the wave functions of the D and the ${ }_{\Lambda}^{3} \mathrm{H}$. For ${ }^{3} \mathrm{He}$ we maintain the Gaussian ansatz of Sec. III B.

\section{Deuteron wave function}

A more accurate parametrization of the $\mathrm{D}$ wave function, that should be used instead of the Gaussian ansatz for quantitative analyses, is given by the Hulthen formula:

$$
\phi_{d}(\mathbf{r})=\sqrt{\frac{\alpha \beta(\alpha+\beta)}{2 \pi(\alpha-\beta)^{2}}} \frac{e^{-\alpha|\mathbf{r}|}-e^{-\beta|\mathbf{r}|}}{|\mathbf{r}|} .
$$

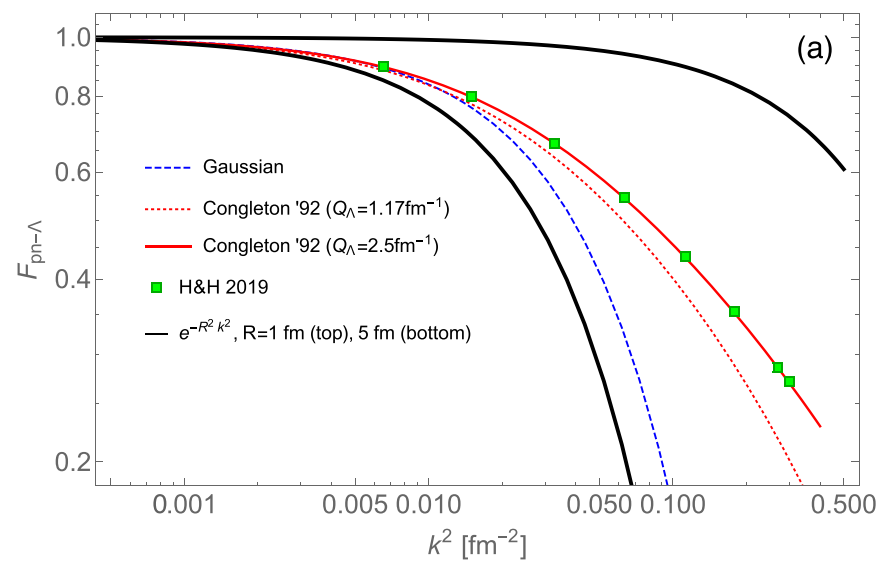

The RMS radius is given by

$$
\begin{aligned}
r_{\mathrm{rms}}^{2} & =\frac{\beta(\alpha+\beta)}{8 \alpha^{2}(\alpha-\beta)^{2}}\left(1-\frac{16 \alpha^{3}}{(\alpha+\beta)^{3}}+\frac{\alpha^{3}}{\beta^{3}}\right) \\
& =\frac{1}{8 \alpha^{2}}\left[1+\frac{3 \alpha}{\beta}+O\left(\frac{\alpha^{2}}{\beta^{2}}\right)\right] .
\end{aligned}
$$

For this parametrization it is assumed that $\beta>\alpha$, so above we expanded in the ratio $(\alpha / \beta)$. For the numerical evaluation we set $\alpha=0.2 \mathrm{fm}^{-1}$ and $\beta=1.56 \mathrm{fm}^{-1}$, reproducing $r_{\text {rms }}=$ $2.13 \mathrm{fm}[51]$. We have checked that using the slightly different values of $\alpha$ and $\beta$ quoted in Ref. [52] gives results that are equal to ours to $5 \%$ accuracy. The form factor Eq. (17) can only be obtained numerically. Amusingly, in the 1D Gaussian source limit Eq. (39) we do not need it because the coalescence factor itself can be obtained analytically [30]:

$$
\begin{aligned}
\mathcal{B}_{2}= & \frac{3}{2 m} \int d^{3} \mathbf{k} e^{-\mathbf{k}^{2} R_{\mathrm{inv}}^{2}} \int d^{3} \mathbf{r}\left|\phi_{d}(\mathbf{r})\right|^{2} e^{-i \mathbf{k r}} \\
= & \frac{3 \pi^{2}}{m R_{\mathrm{inv}}^{2}} \frac{\alpha \beta(\alpha+\beta)}{(\alpha-\beta)^{2}}\left(e^{4 \alpha^{2} R_{\mathrm{inv}}^{2}} \operatorname{erfc}\left(2 \alpha R_{\mathrm{inv}}\right)\right. \\
& \left.-2 e^{(\alpha+\beta)^{2} R_{\mathrm{inv}}^{2}} \operatorname{erfc}\left[(\alpha+\beta) R_{\mathrm{inv}}\right]+e^{4 \beta^{2} R_{\mathrm{inv}}^{2}} \operatorname{erfc}\left(2 \beta R_{\mathrm{inv}}\right)\right) .
\end{aligned}
$$

The coalescence factor $\mathcal{B}_{2}$ is shown in Fig. 1 . The solid line shows the prediction of Eq. (54), obtained for the Hulthen wave function. For comparison, the dashed line shows the less accurate Gaussian wave function prediction of Eq. (49). For simplicity here we use the isotropic 1D Gaussian source model with $R_{o}=R_{s}=R_{l}=R_{\text {inv }}$ and $\mathcal{N}_{2}=1$. As seen in Fig. 1, the largest sensitivity to the effect of the wave function is for measurements at small $R_{\mathrm{inv}} \approx 1 \mathrm{fm}$, which are achievable in $p p$ or $p \mathrm{~Pb}$ collisions [20].

To use the 3D source Eq. (41), the coalescence factor needs to be calculated numerically from Eq. (18) or (15).

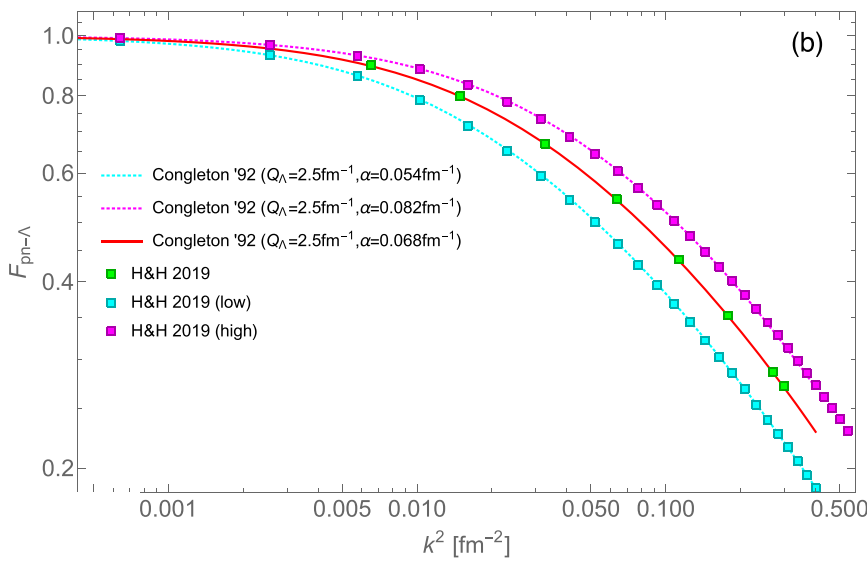

FIG. 2. Hypertriton $p n-\Lambda$ form factor. Green squares show the result from the three-body calculation of Hildenbrand and Hammer [21]. (a) Dotted and solid red line shows the effective two-body calculation of Congleton [53] for different values of their $Q_{\Lambda}$ parameter. The blue dashed line shows a Gaussian approximation with the same charge radius as that found in Ref. [21]. For comparison, thick solid black lines show exponential factors with scale radii $R=1 \mathrm{fm}$ (top) and $5 \mathrm{fm}$ (bottom), respectively. (b) Cyan and magenta markers show a numerical calculation delimiting the uncertainty due to the ${ }_{\Lambda}^{3} \mathrm{H}$ binding energy, as implemented in Ref. [21]. 


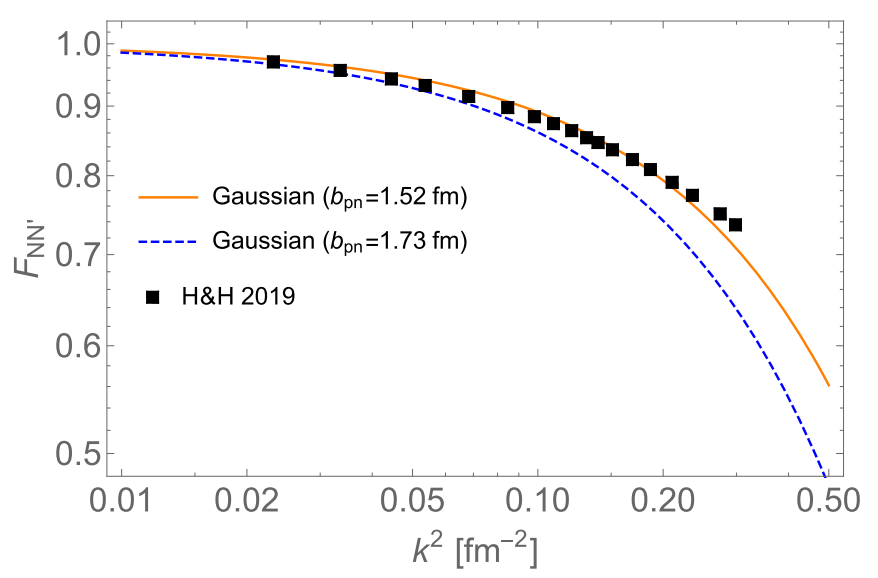

FIG. 3. Hypertriton $p n$ form factor. Black squares show the result from the three-body calculation of Hildenbrand and Hammer [21]. Solid orange and dashed blue lines show a Gaussian approximation à $l a$ Eq. (48) with $b_{p n}=1.52 \mathrm{fm}$ and $b_{p n}=1.73 \mathrm{fm}$, respectively.

\section{Hypertriton wave function}

Hildenbrand and Hammer [21] reported a three-body theoretical calculation of the ${ }_{\Lambda}^{3} \mathrm{H}$ wave function. Green squares in Fig. 2(a) show the projected $p n-\Lambda$ form factor obtained in that work. It is well reproduced by the two-body calculation of Congleton [53], shown by solid red line, provided we adjust the $Q_{\Lambda}$ parameter of [53] from $Q_{\Lambda}=1.17 \mathrm{fm}^{-1}$ in the original paper to $Q_{\Lambda}=2.5 \mathrm{fm}^{-1}$. For simplicity we therefore consider the effective $p n-\Lambda$ wave function from [53], given in momentum space by

$$
\hat{\phi}_{{ }^{3} \mathrm{H}(\Lambda d)}(\mathbf{q})=A \frac{e^{-\frac{\mathbf{q}^{2}}{Q_{\Lambda}^{2}}}}{\mathbf{q}^{2}+\alpha_{\Lambda}^{2}}=\int d^{3} \mathbf{r}_{\Lambda} e^{-i \mathbf{r}_{\Lambda} \mathbf{q}_{\Lambda}} \phi_{{ }_{\Lambda} \mathrm{H}(\Lambda d)}\left(\mathbf{r}_{\Lambda}\right),
$$

where the normalization constant $A$ is defined such that $\int d^{3} \mathbf{r}_{\Lambda}\left|\phi_{\Lambda}^{3} \mathrm{H}(\Lambda d)\left(\mathbf{r}_{\Lambda}\right)\right|^{2}=\int \frac{d^{3} \mathbf{q}}{(2 \pi)^{3}}\left|\hat{\phi}_{{ }^{3}} \mathrm{H}(\Lambda d)(\mathbf{q})\right|^{2}=1$. Using the $p n-\Lambda$ wave function of [53], with $Q_{\Lambda}=2.5 \mathrm{fm}^{-1}$ and $\alpha=$ $0.068 \mathrm{fm}^{-1}$, we can calculate the integrals in Eq. (34) or (30) numerically.

In Fig. 2(b) we show numerical calculations from [21], delimiting the uncertainty due to the ${ }_{\Lambda}^{3} \mathrm{H}$ binding energy. ${ }^{16} \mathrm{We}$ find that the lower (upper) range for the form factor is well fitted again by Congleton's formula, with $Q_{\Lambda}=2.5 \mathrm{fm}^{-1}$ and $\alpha=0.054$ (0.082) $\mathrm{fm}^{-1}$, respectively.

Reference [21] also provided the effective form factor for the $p n$ subsystem. Their numerical result is shown by black squares in Fig. 3. This form factor is reasonably well reproduced by a Gaussian of the form given by Eq. (48), with $b_{p n}=1.52 \mathrm{fm}$ (orange solid line). This can be compared with the low-k $\mathbf{k}^{2}$ expansion of the form factor, which would lead to $b_{p n}\left(\right.$ low-k $\mathbf{k}^{2}$ fit $)=1.73 \mathrm{fm}$ as noted in Sec. III B. Given this discussion, we can maintain the Gaussian ansatz of the $p n$ factor in Eq. (48), setting $b_{p n}=1.52 \mathrm{fm}$.

\footnotetext{
${ }^{16}$ We are grateful to Fabian Hildenbrand for providing this calculation to us.
}

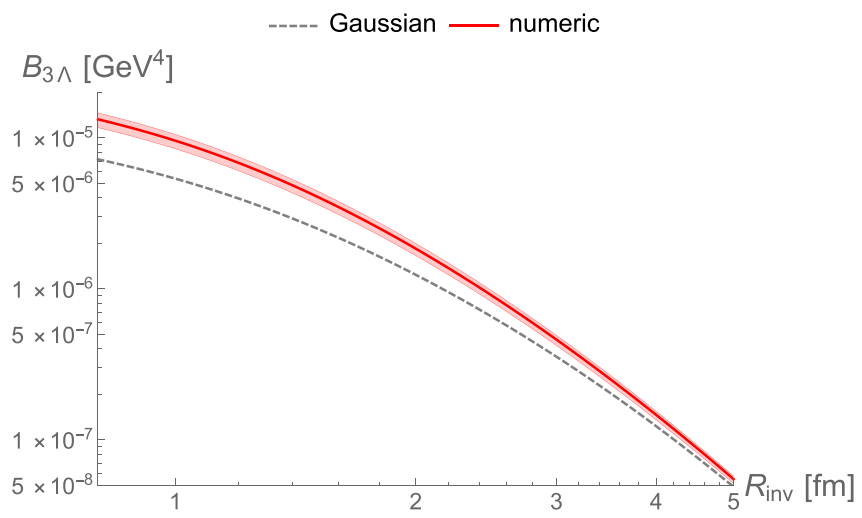

FIG. 4. Hypertriton coalescence factor $\mathcal{B}_{3 \Lambda}$, calculated in the isotropic (1D) Gaussian source model, showing the difference between the Gaussian (dashed grey) and the more realistic numerical (red) wave function parametrizations. We set $\mathcal{N}_{3 \Lambda}=1$.

In Fig. 4 we plot $\mathcal{B}_{3 \Lambda}$ vs $R_{\text {inv }}$, showing the difference between the Gaussian (dashed grey) and the more realistic numerical (red) wave function parametrizations. The shaded band around the numerical result reflects the wave function uncertainty, as depicted in the right panel of Fig. 2.

\section{COMPARISON WITH DATA}

\section{A. Deuteron and ${ }^{3} \mathrm{He}$}

Figure 5 shows the theoretical prediction for $\mathcal{B}_{2}$ (a) and $\mathcal{B}_{3}$ (b), calculated as function of the 1D femtoscopy parameter $R_{\text {inv }}$ using Eq. (54) based on the Hulthen wave function for D and Eq. (50) based on the Gaussian wave function, for ${ }^{3} \mathrm{He}$, with $R_{o}=R_{s}=R_{l}=R_{\text {inv }}$. For $\mathcal{B}_{2}$, the calculation shown by the solid line assumes $\mathcal{N}_{2}=1$, while the dashed lines assumes $\mathcal{N}_{2}=0.5$. For $\mathcal{B}_{3}$ we use $\mathcal{N}_{3}=\mathcal{N}_{2}^{\frac{3}{2}}$.

Our choice to illustrate the results for $\mathcal{N}_{2}$ in the range $(0.5-1)$ is motivated as follows. For large $R_{\text {inv }}$ we can estimate $\mathcal{N}_{2}$ from the $\mathrm{PbPb}$ collisions of [42], obtained as the sum $\mathcal{N}_{2} \approx \lambda^{(p p)}+\lambda^{(p \Lambda)}$ in the notation of that paper. ${ }^{17}$ This suggests $\mathcal{N}_{2} \approx(0.3-0.7)$, roughly consistent with our illustrative values. For small $R_{\text {inv }}$, a measurement of the chaoticity parameter $\lambda \approx 0.5$ exists from kaon femtoscopy [54], consistent with our lower value for $\mathcal{N}_{2}$.

Based on our analysis in Appendix B we can expect that for the $p_{t} \lesssim 1.5 \mathrm{GeV}$ values, in which the cluster data in Fig. 5 are given, our use of the simplistic 1D source parametrization should cause us to overestimate a more accurate $3 \mathrm{D}$ prediction (not available to us, as the experiments reported 1D

\footnotetext{
${ }^{17}$ The $\lambda^{(i j)}$ parameters were introduced in Ref. [42] to account for feed-down from weak decays. As recalled in Appendix A 1, while weak decays do indeed act to dilute the observed femtoscopic correlation signal, their effect is, in principle, distinct from the genuine femtoscopic quantity $\mathcal{N}_{2}$. However, Ref. [42] let the $\lambda^{(i j)}$ float in the fit to the data, rather than imposing $\sum \lambda^{(i j)}=1$ as would apply for feed-down probabilities. In doing so they provided an effective measurement of $\mathcal{N}_{2}$ [19].
} 

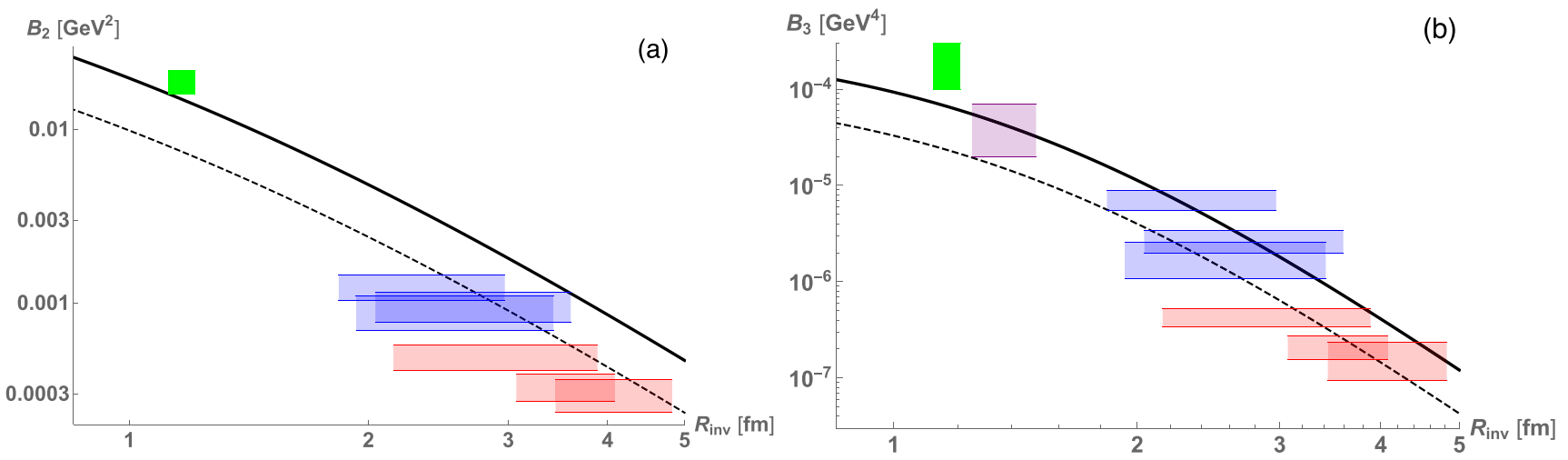

FIG. 5. Summary of D and ${ }^{3} \mathrm{He}$ data, reproduced from Ref. [19] with some improvements (see text). (a) $\mathcal{B}_{2}$ vs $R_{\text {inv }}$, using the Hulthen wave function for $\mathrm{D}$. The solid (dashed) line shows the result for $\mathcal{N}_{2}=1(0.5)$. (b) $\mathcal{B}_{3}$ vs $R_{\text {inv }}$, using $\mathcal{N}_{3}=\mathcal{N}_{2}^{\frac{3}{2}}$.

femtoscopy fits only) by $\lesssim 20 \%$ or so. We do not include this uncertainty in the plot. It adds up other sources of systematic uncertainty, expected to be roughly at a similar level (with unknown signs), due to the smoothness, equal-time, and factorization approximations (the latter relevant for ${ }^{3} \mathrm{He}$ only).

The comparison to experimental data is as follows. The red horizontal bands in Fig. 5 show experimental coalescence factor measurements for $\mathrm{PbPb}$ at $(0-10 \%)$ (for $\left.\mathcal{B}_{2}\right)$ and $(0$ $20 \%$ ) (for $\mathcal{B}_{3}$ ) centrality classes [55]. Each of the three red bands corresponds to a different bin in $m_{t}$, among the three bins shown in the femtoscopy $R_{\text {inv }}$ measurement [42]. The blue horizontal bands show the result for the (20-40\%) (for $\mathcal{B}_{2}$ ) and (20-80\%) (for $\mathcal{B}_{3}$ ) events, respectively, again from [55].

The green band shows the result for $p$ - $p$ collisions [56]. ${ }^{18}$

For ${ }^{3} \mathrm{He}$ we can also add a crudely estimated data point for $p \mathrm{~Pb}$ collisions. To do so, we combine the 1D femtoscopy $R_{\text {inv }}$ measurement of kaon femtoscopy reported in Ref. [57] with the ${ }^{3} \mathrm{He}$ measurement of [58]. To approximately match $m_{t}$ between the data sets, we use here the highest $k_{t}$ bin in Ref. [57] and the lowest $p_{t}$ bin in Ref. [58]. We use the (0-20\%) multiplicity class from [57], joining together the $(0-10 \%)$ and $(10-20 \%) \mathcal{B}_{3}$ data from [58]. The result is shown in purple in Fig. 5(b).

As should be clear by now, a main uncertainty in the theory prediction shown in Fig. 5 is related to the determination of the parameters $\mathcal{N}_{2}$ and $\mathcal{N}_{3}$. For $\mathcal{N}_{3}$ we have no direct measurements, as such data would require three-proton femtoscopy. In Fig. 5 we bypassed this by assuming $\mathcal{N}_{3}=\mathcal{N}_{2}^{\frac{3}{2}}$. Addressing this issue experimentally would be challenging, and we do not know of a model-independent way to estimate the associated theory uncertainty. Assessing the uncertainty within specific HXS models, along the lines of Appendix B, may be warranted in future work.

Another obvious difficulty is due to the need to construct Fig. 5 patchwise from data at different, often only partially overlapping, multiplicity class and $p_{t}$ or $m_{t}$ bins. A

\footnotetext{
${ }^{18}$ We thank Bhawani Singh and the Fabbietti TUM group for pointing out a typo in the plot of the $\mathcal{B}_{2}$ data for $p p$ collisions in Ref. [19].
}

dedicated experimental analysis combining femtoscopy and cluster yields would solve this problem.

Altogether, Fig. 5 shows that coalescence is roughly consistent with the $\mathrm{D}$ and ${ }^{3} \mathrm{He}$ data for systems ranging from $p p$ to $p \mathrm{~Pb}$ and $\mathrm{PbPb}$ at different regions of $p_{t}$ and at different multiplicity classes. This comparison spans a dynamical range of about a factor of 30 for $\mathcal{B}_{2}$ and a factor of $10^{3}$ for $\mathcal{B}_{3}$. While, as we discussed, there are theoretical and experimental uncertainties, there are no free parameters once femtoscopy calibrates the computation. From this point of view, the usual claim to fame of the SHM [4], to describe the yields of nuclei across many orders of magnitude, is seen to be comparably well applicable to coalescence.

Having said that, it is worth highlighting that for $p p$ collisions the experimental coalescence factors for both $\mathrm{D}$ and ${ }^{3} \mathrm{He}$ are found to be higher than the coalescence prediction. Depending mainly on the precision with which $\mathcal{N}_{2}$ is determined, but also on possible systematic uncertainties related to different event classes entering the femtoscopy and cluster measurements, the discrepancy could be as much as a factor of 2 for $\mathrm{D}$ and a factor of 4 for ${ }^{3} \mathrm{He}$. We think that this situation is strong motivation for a joint experimental analysis of coalescence and femtoscopy in small systems.

\section{B. Hypertriton}

Reference [59] reported a measurement of ${ }_{\Lambda}^{3} \mathrm{H}$ in $\mathrm{PbPb}$ collisions. After some interpolations, we extract the measured $\mathcal{B}_{3 \Lambda}$ from the three $p_{t}$ bins in the left panel of their Fig. 7. For the bins $^{19} p_{t} \approx(0.66-1.34),(1.34-2)$, and (2-2.34) $\mathrm{GeV}$ we find $\mathcal{B}_{3 \Lambda} \approx(7.3-20) \times 10^{-8},(1-4.2) \times 10^{-7}$, and $(1.7-12) \times 10^{-5} \mathrm{GeV}^{4}$, respectively. The multiplicity class is (0-10\%). For femtoscopic data, we have Ref. [42] with $R_{\text {inv }}$ and $\mathcal{N}_{2}$ measured in the same multiplicity class, but binned in $m_{t}$ rather than $p_{t}$ (see Figs. 7 and 8 in Ref. [42]). We can match the first low $p_{t}$ bin of [59] into the $m_{t}$ range covered in Ref. [42]. We also consider the second $p_{t}$ bin of [59] that somewhat overshoots the coverage of the last $m_{t}$ bin in Ref. [42]. With

\footnotetext{
${ }^{19}$ Our $p_{t}$ is corresponds to $p_{t} / A$ as defined in Ref. [59].
} 


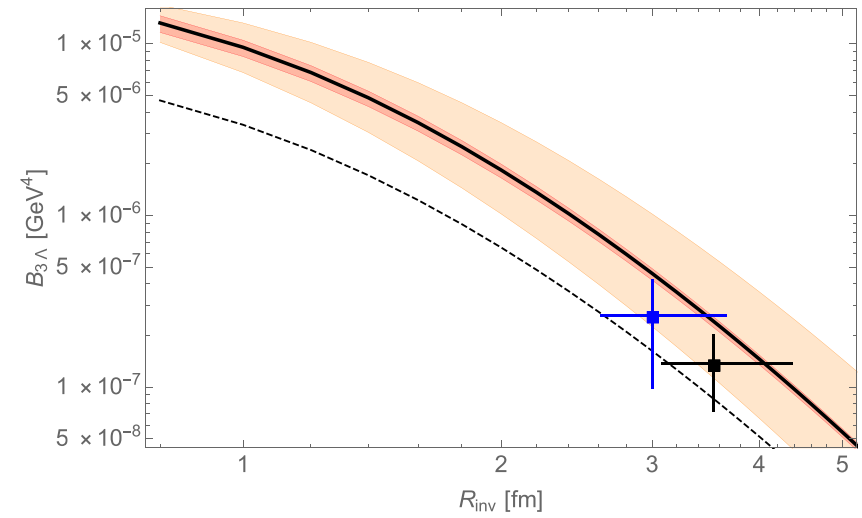

FIG. 6. $\mathcal{B}_{3 \Lambda}$ vs the $1 \mathrm{D}$ femtoscopic radius $R_{\text {inv }}$, showing the coalescence prediction for the numerical wave function of Sec. III C 2. The width of the red band reflects the wave function uncertainty as derived from the calculations of [21], while the orange band reflects the $R_{\text {inv }}$ uncertainty (see text). The solid (dashed) line shows the result for $\mathcal{N}_{3 \Lambda}=\mathcal{N}_{2}^{\frac{3}{2}}$ with $\mathcal{N}_{2}=1(0.5)$.

some interpolation (and, for the second bin, extrapolation) of the results from [42], we obtain the corresponding estimated ranges in $R_{\text {inv }}$. The result is shown by markers in Fig. 6 .

Once again, to plot the theory prediction, we need an estimate of $\mathcal{N}_{3 \Lambda}$. For definiteness we present the results using $\mathcal{N}_{3 \Lambda}=\mathcal{N}_{2}^{\frac{3}{2}}$, with $\mathcal{N}_{2}=1$ (solid line) and $\mathcal{N}_{2}=0.5$ (dashed line). We would like to illustrate the wave function uncertainty, as well as the uncertainty associated with determining $R_{\text {inv }}$ from $p p$ vs $\mathrm{p} \Lambda$ or $\Lambda \Lambda$ correlations (mentioned earlier in Sec. III A). The result of varying the numerical wave function in the range corresponding to the right panel of Fig. 2 is shown by the red band. To implement the $R_{\text {inv }}$ uncertainty, an orange band shows the effect of shifting the $R_{\text {inv }}$ argument, entering the $\mathcal{B}_{3 \Lambda}$ computation, by $\pm 20 \%$ with respect to the $R_{\text {inv }}$ value on the $x$ axis.

We can try to bypass some of the uncertainty associated with the determination of $\mathcal{N}_{3 \Lambda}$ as follows. If $\mathcal{N}_{3} \approx \lambda_{3 \Lambda}$, then the uncertainty associated with $\mathcal{N}_{3 \Lambda}$ may cancel in the ratio ${ }^{20}$

$$
S_{3}=\frac{\mathcal{B}_{3 \Lambda}}{\mathcal{B}_{3}} .
$$

For the first $p_{t}$ bin of [59] we find $\mathcal{B}_{3} \approx(1.4-2.7) \times$ $10^{-7} \mathrm{GeV}^{4}$, and for the second bin $\mathcal{B}_{3} \approx(3.3-6.9) \times$ $10^{-7} \mathrm{GeV}^{4}$. We could, in principle, combine this directly with $\mathcal{B}_{3 \Lambda}$ to extract the measured $S_{3}$. However, this would ignore the fact that some of the experimental uncertainty involved in deriving $\mathcal{B}_{3}$ and $\mathcal{B}_{3 \Lambda}$ could cancel out in the ratio. Instead, we therefore adopt the following procedure. Taking the hypertriton spectrum from [59] (averaging the ${ }_{\Lambda}^{3} \mathrm{H}$ and ${ }_{\Lambda}^{3} \mathrm{H}$ results), we divide by the ${ }^{3} \mathrm{He}$ spectrum from [55] and scale by the $\Lambda / p$ ratio taken from $[60,61]$, evaluated in the corresponding $p_{t}$ interval. The result is shown by markers in Fig. 7 .

\footnotetext{
${ }^{20}$ Not to be confused with the three-particle normalized source, $\mathcal{S}_{3}$, defined in Sec. II B 2.
}

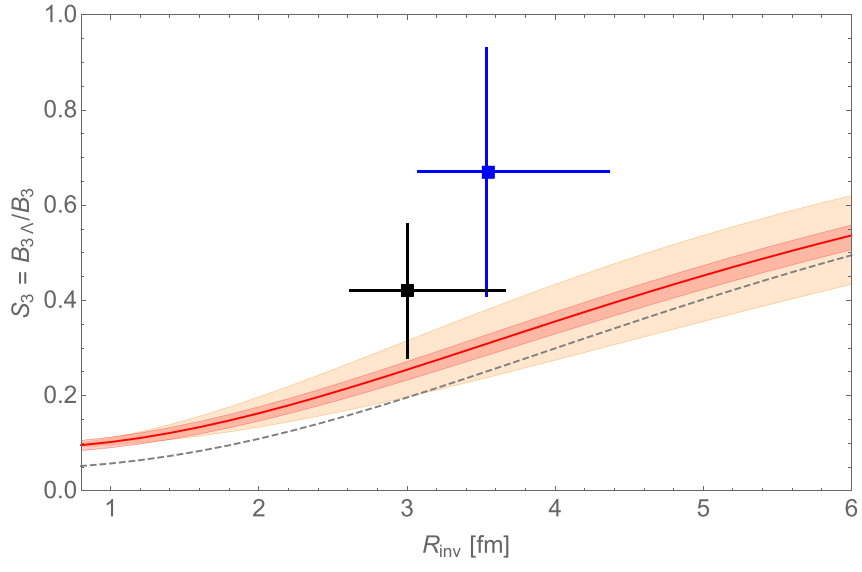

FIG. 7. $S_{3}$ vs $R_{\text {inv }}$ predicted in the coalescence model compared with data.

The theoretical prediction for $S_{3}$, using the numerical ${ }_{\Lambda}^{3} \mathrm{H}$ wave function of Sec. III C 2, is shown by the red band in Fig. 7 with the band width determined by the wave function uncertainty. The effect of varying $R_{\text {inv }}$ by $\pm 20 \%$ is shown by the orange band. For comparison we also show the Gaussian approximation with a grey dashed line. The coalescence prediction is somewhat below the data, with tension at the $2 \sigma$ level. ${ }^{21}$ It is clear from Fig. 7 that a more precise experimental measurement of $S_{3}$ in conjunction with femtoscopy is a promising observable to exclude (or support) the framework. At small $R_{\text {inv }}, S_{3}$ is predicted to be much below unity. Neither the ${ }_{\Lambda}^{3} \mathrm{H}$ wave function uncertainty nor the details of working with a $1 \mathrm{D}$ vs $3 \mathrm{D}$ femtoscopic source parametrization are expected to enable, e.g., $S_{3}>0.2$ at $R_{\text {inv }} \approx 1 \mathrm{fm}$, characteristic for $p p$ collisions.

\section{DISCUSSION AND SUMMARY}

The formation of nuclei by coalescence and femtoscopic [or Hanbury Brown-Twiss (HBT)] correlations between continuum proton pairs are two manifestations of final state interactions (FSI) that "dress" an underlying high-excitation state (HXS) produced in hadronic collisions. In Sec. II, starting from a relativistic quantum field theoretic (QFT) computation, we recalled how the effective quantum mechanical (QM) description of the dynamics of pairs that are nonrelativistic in the pair rest frame (PRF) gives rise to the usual femtoscopy formalism, with which a two-particle source, characterizing the HXS, can be measured. The same twoparticle source enters the coalescence formula for deuteron formation. Much of our analysis was based on the formalism reviewed by Lednicky [18]; our contribution was to reduce this formalism to observationally accessible language in the context of nuclear clusters. In both cases-femtoscopy and

\footnotetext{
${ }^{21}$ To quantify the significance of the tension more precisely we would need to combine the two data points, which very much overlap in $R_{\text {inv }}$. We prefer to leave these details to a dedicated experimental analysis.
} 
coalescence-effective QM holds for $\mathbf{q}^{\mathbf{2}} \ll \mathbf{m}^{\mathbf{2}}$, where $\mathbf{q}$ is the PRF momentum difference and $m$ is the nucleon mass; and up to corrections of order $0.2 \frac{1 \mathrm{fm}}{\mathbf{x}}$, where $\mathbf{x}$ is the average PRF characteristic distance between the nucleons in the HXS. Although it should not be trusted beyond $O(10 \%)$ accuracy, ${ }^{22}$ the QM limit is useful for deriving simple physically transparent formulas and should be sufficient for ruling out (or supporting) coalescence as the origin of nuclei in hadronic collisions. In principle, while we did not pursue this route, the QM approximation can be avoided if one works directly with the relativistic FSI Bethe-Salpeter amplitudes.

Coalescence after kinetic freeze-out must occur at some level in hadronic collisions, but this does not mean that it is necessarily the dominant origin of clusters. The key assumption made by the coalescence model is that the long-range action of FSI can be factored out of an underlying short-range HXS dynamics. The level of accuracy of this factorization is not obvious. To some extent, the apparent success of femtoscopy analyses [20,42,43] in reconstructing a fit of the two-nucleon source, using sophisticated physical FSI calculations, supports the coalescence framework. But we feel that this is not yet fully convincing: while the femtoscopy analyses $[20,42,43]$ reported measurements of the two-nucleon source with a stated precision of $O(10 \%)$, they did so using a $1 \mathrm{D}$ Gaussian source fit. ${ }^{23}$ However, the true underlying source is not expected to be 1D; in fact, at least for mean laboratory frame momentum $\mathbf{p} \gtrsim m$, it must be anisotropic at the $O(1)$ level-as seen in the PRF-due to Lorentz contraction. We can add to this the uncertainty related to the normalization or chaoticity parameter $\mathcal{N}_{2}$, which existing analyses typically did not consider in the fit (an exception is Ref. [42], that may have accessed $\mathcal{N}_{2}$ effectively and indirectly by allowing the feed-down probabilities to vary in the fit). Altogether, it is clear that higher statistics femtoscopy analyses have an important future role to play [63] in establishing to what extent true physical HXS information is revealed in femtoscopy. Our work highlights the importance of this question also to the origin of clusters.

Keeping the caveats above in mind, the main point of our work is to establish the coalescence/femtoscopy framework as a means to test coalescence by grounding the coalescence predictions with femtoscopy information. The coalescence-correlations relation between femtoscopy and deuteron formation, summarized by Eqs. (14) and (15) [or equivalently Eqs. (16) and (18) in momentum space], was derived first in Ref. [19] in the QM limit. The relation is very general and model independent. As shown in Ref. [19], it is consistent with the early model-dependent derivation of [15] (the latter, however, was specialized to a particular model of hydrodynamical flow and was limited to small $p_{t}$ ). It is also consistent with the body of work by Mrowczynski [28-34].

The connection of femtoscopy with deuteron formation does not rely on density matrix factorization. To derive for-

\footnotetext{
${ }^{22}$ Making some interesting proposed $O(10 \%)$ tests potentially challenging $[45,62]$.

${ }^{23}$ This is true also for [20], which assumed a 1D core source and modulated it by strong resonance decays.
}

mulas for three-body clusters, though, factorization is needed. Assuming factorization we derived the formulas for ${ }_{\Lambda}^{3} \mathrm{H}$ and ${ }^{3} \mathrm{He}$, summarized in Eqs. (30) and (31) [or Eqs. (34) and (35) in momentum space]. As an aside, we note that the derivation does not leave room for the confusion between " $p n \Lambda$ channel" and "D $\Lambda$ channel" advocated in Refs. [22,23] for ${ }_{\Lambda}^{3} \mathrm{H}$. Threebody coalescence comes from three-body FSI and is captured by a single formula, involving the three-body nucleus wave function.

In Sec. III we combined formulas for the two-nucleon source, constrained by experimental femtoscopy fits, with nuclear wave functions to obtain expressions for coalescence factors that can be compared to data. Exploring different forms for the wave function of ${ }_{\Lambda}^{3} \mathrm{H}$, we showed that, although a full numerical representation of the wave function deviates significantly from a Gaussian, the numerical impact on the coalescence factor is modest, about a factor of 2 (with the more realistic numerical wave function predicting a higher coalescence factor). Our calculations allow for different scales in the wave functions of three-body states. This is particularly important for ${ }_{\Lambda}^{3} \mathrm{H}$ where the $p n-\Lambda$ factor is significantly more extended than the effective $p n$ factor. Some earlier implementations [24] of the coalescence-correlations relation for ${ }_{\Lambda}^{3} \mathrm{H}$ did not account for this fact.

Our calculations also account for the anisotropic shape of the two- and three-nucleon source describing the HXS. As we illustrate in Appendix B, the two-particle source is expected to be truly anisotropic in nature, especially at large $p_{t}$.

In Sec. IV we compared our theoretical predictions to data. Figure 5 shows that coalescence, calibrated by femtoscopy, is consistent at the $O(1)$ level with the $p_{t}$-differential yields of $\mathrm{D}$ and ${ }^{3} \mathrm{He}$ for systems ranging from $p p, p \mathrm{~Pb}$ to $\mathrm{PbPb}$ and across different centralities. This comparison spans a dynamical range of 30 for $\mathcal{B}_{2}$ and $10^{3}$ for $\mathcal{B}_{3}$. Some tension, at the $2 \sigma$ level or so, is seen for $p p$ in both of the $\mathrm{D}$ and ${ }^{3} \mathrm{He}$ yields. This situation gives strong motivation for a dedicated experimental analysis, studying femtoscopy and cluster yields side by side in the same data set under the same kinematic conventions and cuts. On the femtoscopy side, we urge the experiments to report femtoscopy measurements adding the chaoticity parameter $\mathcal{N}_{2}$ to the fit. In addition, as much as statistics permits, measurements of the 3D source (in the out-side-long parametrization) would be preferred over 1D measurements of $R_{\text {inv }}$. Such combined experimental analysis could zoom in on the coalescence-correlations prediction beyond the $O(1)$ level by which it can currently be tested. This could, in principle, sharpen tensions with $\mathrm{D}$ and/or ${ }^{3} \mathrm{He}$ data where they are currently difficult to establish conclusively.

Hypertriton ${ }_{\Lambda}^{3} \mathrm{H}$ is known as a sensitive test of coalescence because of its large size, suggesting that the QM factor discriminating coalescence from the statistical hadronization model (SHM) should be small and discernible. Our calculation, depicted in Fig. 6, shows that the current measurements of $\mathcal{B}_{3 \Lambda}$ in $\mathrm{PbPb}$ collisions are consistent with the coalescence prediction. The observable $S_{3}=\mathcal{B}_{3 \Lambda} / \mathcal{B}_{3}$ may be more robust than $\mathcal{B}_{3 \Lambda}$, because some experimental and theoretical uncertainties may cancel in the ratio. We compare our calculation of $S_{3}$ with the data in Fig. 7 , finding some tension: the $S_{3}$ 
data tend to be somewhat higher than coalescence predicts. This discrepancy is not (yet) very significant, around $2 \sigma$. A higher statistics measurement, and measurements in small systems like $p p, \mathrm{pPb}$, or low multiplicity $\mathrm{PbPb}$ collisions-in short, systems with small femtoscopic radius-would provide a critical test of coalescence.

\section{ACKNOWLEDGMENTS}

We thank Fabian Hildenbrand for providing us with numerical calculations of the ${ }_{\Lambda}^{3} \mathrm{H}$ wave function, the Fabbietti TUM group, Stanislaw Mrowczynski, Urs Wiedemann, and the participants of the CERN workshop "Origin of nuclear clusters" for insightful discussions and Nitsan Bar and Yossi Nir for comments on the manuscript.

\section{APPENDIX A: CORRECTIONS TO THE GAUSSIAN SOURCE APPROXIMATION}

The Gaussian source considered in Sec. III A cannot be exact. It is therefore important to estimate the uncertainty in the coalescence calculation, that comes about if this approximation is used. In this section we consider mechanisms that violate the Gaussian source approximation and estimate their quantitative impact.

\section{Feed-down from weak decays and source chaoticity}

In comparing theory to data one should account for the effect of feed-down from weak decay, where a particle that is emitted from the HXS as $\Lambda$ or $\Sigma$ decays into a proton in the detector (see [50] for a parallel discussion for pions). The decay vertex is displaced by $O(1 \mathrm{~cm})$ from the HXS, which means that the FSI relevant for two-particle correlation are those involving $\Lambda$ or $\Sigma$. Reference [20] estimated that a fraction $\alpha_{p} \approx 0.82$ of detected protons in their analysis originate from a genuine emitted proton, while a fraction $\alpha_{\Lambda} \approx$ 0.12 and a fraction $\alpha_{\Sigma} \approx 0.06$ of detected protons originate from an emitted $\Lambda$ or $\Sigma$, respectively. ${ }^{24}$ Neglecting particle misidentification (which add up to about $1 \%$ in Ref. [20]) we have $\alpha_{p} \approx 1-\alpha_{\Lambda}-\alpha_{\Sigma}$. Thus, iffemtoscopic correlations are ignored, a fraction $\lambda^{(p p)} \approx\left(1-\alpha_{\Lambda}-\alpha_{\Sigma}\right)^{2} \approx 0.7$ of detected $p p$ pairs come from genuine emitted $p p$, while, for example, a fraction $\lambda^{(p \Lambda)} \approx 2 \alpha_{\Lambda}\left(1-\alpha_{\Lambda}-\alpha_{\Sigma}\right) \approx 0.2$ of detected $p p$ pairs come from an emitted $\mathrm{p} \Lambda$ pair, etc.

In constructing the observable two-proton correlation, Refs. [20,42,43] divided the measured proton pair spectrum by the spectrum of pairs from uncorrelated mixed events, without feed-down subtraction. Then, in fitting a model of the correlation to the data, the correlation function was split into a part coming from genuine emitted $p p$ pairs and parts coming from emitted $\mathrm{p} \Lambda, \Lambda \Lambda, p \Sigma$, and $\Sigma \Sigma$ pairs, using the relevant

\footnotetext{
${ }^{24}$ The feed-down fractions in Ref. [20] were allowed to vary by $20 \%$ as part of the systematic uncertainty estimate. Indeed, these fractions were different by about $20 \%$ in Ref. [43], although this may be in part due to different experimental kinematical cuts and selection criteria.
}

FSI for each part: ${ }^{25}$

$$
C^{\text {model }}(\mathbf{p}, \mathbf{q})=1+\sum_{i=p p, p \Lambda, \ldots} \lambda^{(i)}\left[C^{(i)}(\mathbf{p}, \mathbf{q})-1\right],
$$

with the channel-specific weights and correlation functions

$$
C^{(i)}(\mathbf{p}, \mathbf{q})=\sum_{s} w_{s} \int d^{3} \mathbf{r} \mathcal{S}_{2}^{(i)}(\mathbf{r})\left|\phi_{s, q}^{(i)}(\mathbf{r})\right|^{2} .
$$

Here, for example, for $i=p \Lambda$ the wave function at spin channel $s$ is given by $\phi_{s, q}^{(p \Lambda)}(\mathbf{r})$, etc. In Ref. [20], the nucleon source functions $\mathcal{S}_{2}^{(i)}(\mathbf{r})$ were modeled differently for different $i$, to account for strong resonances that have a different characteristic decay range for $p$ and $\Lambda$ daughters. In contrast, Refs. [42,43] assumed a common nucleon source $\mathcal{S}_{2}^{(i)}=\mathcal{S}_{2}$.

As long as the $\lambda^{(i)}$ parameters are correctly calibrated to account for weak decays, a quick check verifies that the correlation functions $C^{(i)}$ in Eq. (A2) do indeed match with the theoretical definition of the same objects as derived in Sec. II A. References [20,43] assumed that this was the case and fixed the numerical values of the $\lambda^{(i)}$ according to the experimentally determined single-proton purity. In contrast, Ref. [42] did not fix the value of $\lambda^{(p p)}$ and $\lambda^{(p \Lambda)}$, but rather considered these parameters as part of the experimental fit. Interestingly, the fit resulted in $\lambda \equiv \lambda^{(p p)}+\lambda^{(p \Lambda)} \approx 0.3-0.7$, significantly less than unity. ${ }^{26}$

Both approaches, [42,54] as well as [20,43], assume in their fit the 1D Gaussian form, Eq. (36), of the source function $\mathcal{S}_{2}(\mathbf{r})[12,19]$. This source function integrates to unity, $\int d^{3} \mathbf{r} \mathcal{S}_{2}(\mathbf{r})=1$, corresponding to $\mathcal{C}_{2}(\mathbf{q}=0)=1$. However, there is no a priori reason to assume that the true $\mathcal{C}_{2}$ (or $\mathcal{S}_{2}$ ) satisfy this normalization exactly. In fact, observing a departure from this normalization in the data could hint, for example, at a violation of the factorization assumption of Sec. II B. In addition, it is well known that if the experimental fit assumes a Gaussian form for $\mathcal{C}_{2}(\mathbf{q})$, but the true $\mathcal{C}_{2}(\mathbf{q})$ is non-Gaussian, then adding an intercept parameter $\mathcal{N}_{2}$ to the fit, as in Eq. (41), can absorb some of the difference. We can interpret the $\lambda$ measurement of [42] as an approximation of the parameter $\mathcal{N}_{2}$, via $\mathcal{N}_{2} \approx \lambda$. In contrast, as Refs. [20,43] fixed the $\lambda$ parameters as feed-down probabilities, these analyses may well have better control on the impact of weak decays in the observed correlation function, but they do not yield an estimate of $\mathcal{N}_{2}$.

An optimal experimental procedure may be to add $\mathcal{N}_{2}$ as a true femtoscopic fit parameter describing the normalization of the source function, while at the same time following the self-consistent feed-down procedure of $[20,43]$.

Considering now the coalescence factor, a direct comparison to the femtoscopy analysis is possible if the single-proton

\footnotetext{
${ }^{25}$ Reference [64] also used a similar formalism in studying baryonantibaryon correlations.

${ }^{26}$ Useful details can be found in Ref. [65]. For comparison, the single-particle purity estimates of $[20,43]$ read $\lambda^{(p p)}+\lambda^{(p \Lambda)} \approx 0.87$ and 0.9 , respectively; the remaining probability being associated mostly with $\mathrm{p} \Sigma$ pairs.
} 

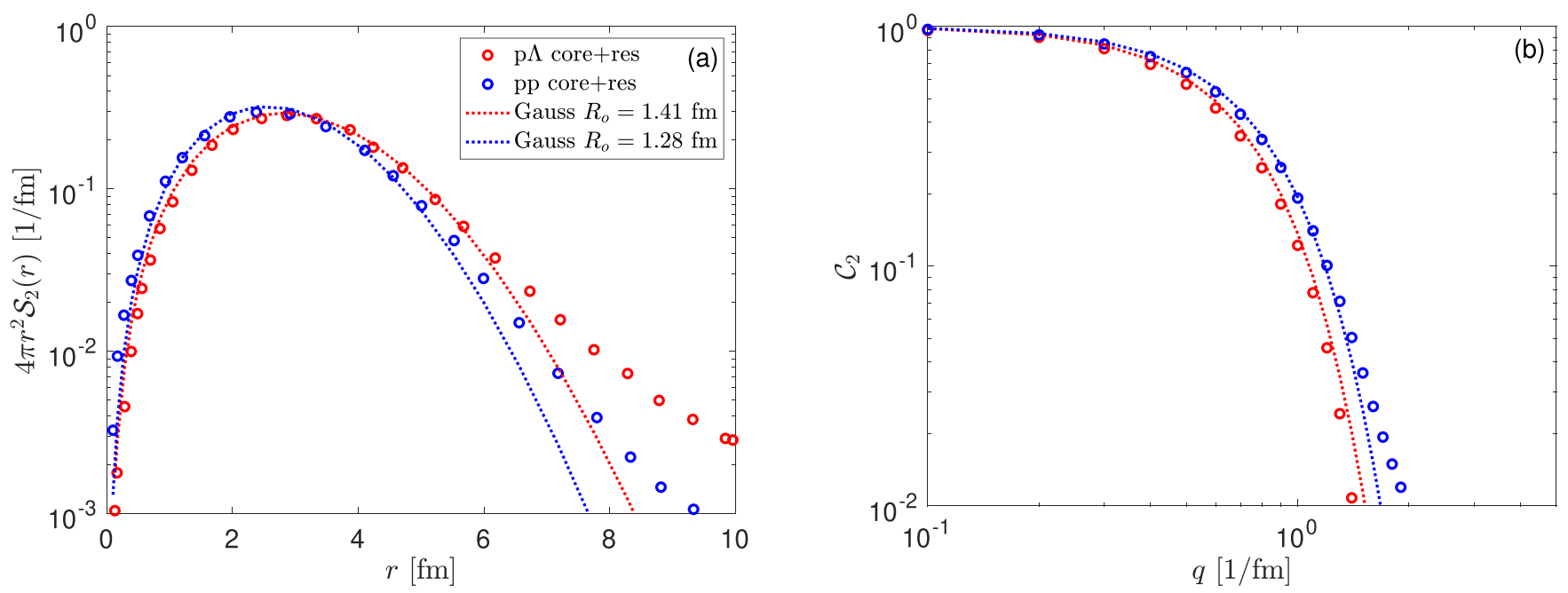

FIG. 8. (a) The source $\mathcal{S}_{2}(\mathbf{r})$, reproduced from the experimental fit result of Fig. 4 in Ref. [20]. (b) $\mathcal{C}_{2}$, the Fourier transform of the sources on the left.

spectrum used in the experimental definition of $\mathcal{B}_{A}$ [e.g., Eq. (10)] is obtained subtracting the weak decay feed-down contributions. With this the coalescence-correlation relation of Eq. (15) or Eq. (18) applies as is. For $\mathcal{B}_{3,3 \Lambda}$, a fully datadriven analysis would require an experimental measurement of $\mathcal{C}_{3}$. In the absence of that, we can roughly estimate that for $\mathcal{N}_{2} \neq 1$ the Gaussian source expressions for $\mathcal{B}_{3,3 \Lambda}$ should be modified by $\mathcal{B}_{3,3 \Lambda} \rightarrow \mathcal{N}_{2}^{\frac{3}{2}} \mathcal{B}_{3,3 \Lambda}[19]$.

Unrelated to weak decays, a correction to the expressions of Sec. III arises from the fact that Ref. [20] actually found different fit results for the sources $\mathcal{S}_{2}(\mathbf{r})$ deduced from $p p$ and $\mathrm{p} \Lambda$ pairs. One physically motivated reason for the difference stems from the different decay range of the strong resonances, that feed into $p$ and $\Lambda$ states in the HXS. The same effect also predicts a non-Gaussian form for $\mathcal{S}_{2}(\mathbf{r})$ [and $\mathcal{C}_{2}(\mathbf{q})$ ], as we discuss next.

\section{Strong resonance decays}

Even if the underlying emission function was an exact Gaussian, the decay of strong resonances with lifetimes of the order of a few fm would distort the effective source. Reference [20] studied this problem for $p p$ and $p \Lambda$ correlations in $p p$ collisions. An isotropic source model including strong resonance decay, which was found in Ref. [20] to give an adequate fit to the correlation data, is reproduced here in Fig. 8(a). The full (non-Gaussian) source is shown by circles, compared with the Gaussian source in dotted lines. Strong resonances lead to a non-Gaussian tail of $\mathcal{S}_{2}(\mathbf{r})$. In Fig. 8(b) we show the $\mathcal{C}_{2}(\mathbf{q})$ curves corresponding to the $\mathcal{S}_{2}(\mathbf{r})$ curves on the left.

Quantitatively, despite the strong resonance contribution, the Gaussian approximation can be seen to give a rather accurate description of the source: the difference between the full non-Gaussian $\mathcal{C}_{2}$ and the Gaussian approximation is smaller than about $10 \%$ throughout the range where $\mathcal{C}_{2}>0.1$. While Ref. [20] dealt with $p p$ initial state, we might expect that in larger systems like $\mathrm{PbPb}$ the relative impact of strong resonance decay could be even less significant.

We emphasize that Ref. [20], which provided the example for our discussion, assumed an underlying Gaussian source which they then deformed by strong resonances. What we learn from this exercise, then, is that if the underlying "genuine" emission function is Gaussian, then the effective two-particle source after strong decays is also consistent with Gaussian to $10 \%$ accuracy, in the range of $\mathbf{r}$ (or, in momentum space, q) that is relevant for coalescence. We stress, however, that while the assumed 1D Gaussian source of [20] was experimentally consistent with femtoscopy data, there is no guarantee that the true physical source is Gaussian. In Appendix B we recall theoretical reasons to expect otherwise.

\section{APPENDIX B: DEVIATIONS FROM AN ISOTROPIC GAUSSIAN SOURCE IN THE PHENOMENOLOGICAL BLAST WAVE MODEL}

Relativistic expansion of the HXS "fireball" is expected to proceed differently along and transverse to the beam line. This unisotropic flow predicts that the source $\mathcal{S}_{2}(\mathbf{r})$ should depend on the direction of $\mathbf{r}$ with respect to the beam line and also with respect to the pair mean momentum vector p. Even if the nucleon source was somehow isotropic in the laboratory frame, it would not be seen as isotropic in the PRF for $\mathbf{p} \neq 0$, due to Lorentz contraction. These effects were not modeled in the experimental analyses of Refs. [20,42,43], that performed fits to the 1D source, Eqs. (39). Here we consider these effects from the point of view of the phenomenological blast wave model (BWM). Our goal is not to argue either in favor of or against the validity of the model, but simply to gain some idea of the possible systematic error associated with adopting the 1D Gaussian source approximation, if that is applied to coalescence calculations via the coalescence-correlation relation.

To recall the BWM, we take the beam line to be along the $\hat{z}$ axis. The space-time coordinates are chosen as $\tau=\sqrt{t^{2}-z^{2}}, \rho=\sqrt{x^{2}+y^{2}}, \eta=\operatorname{arctanh}(z / t)$, 

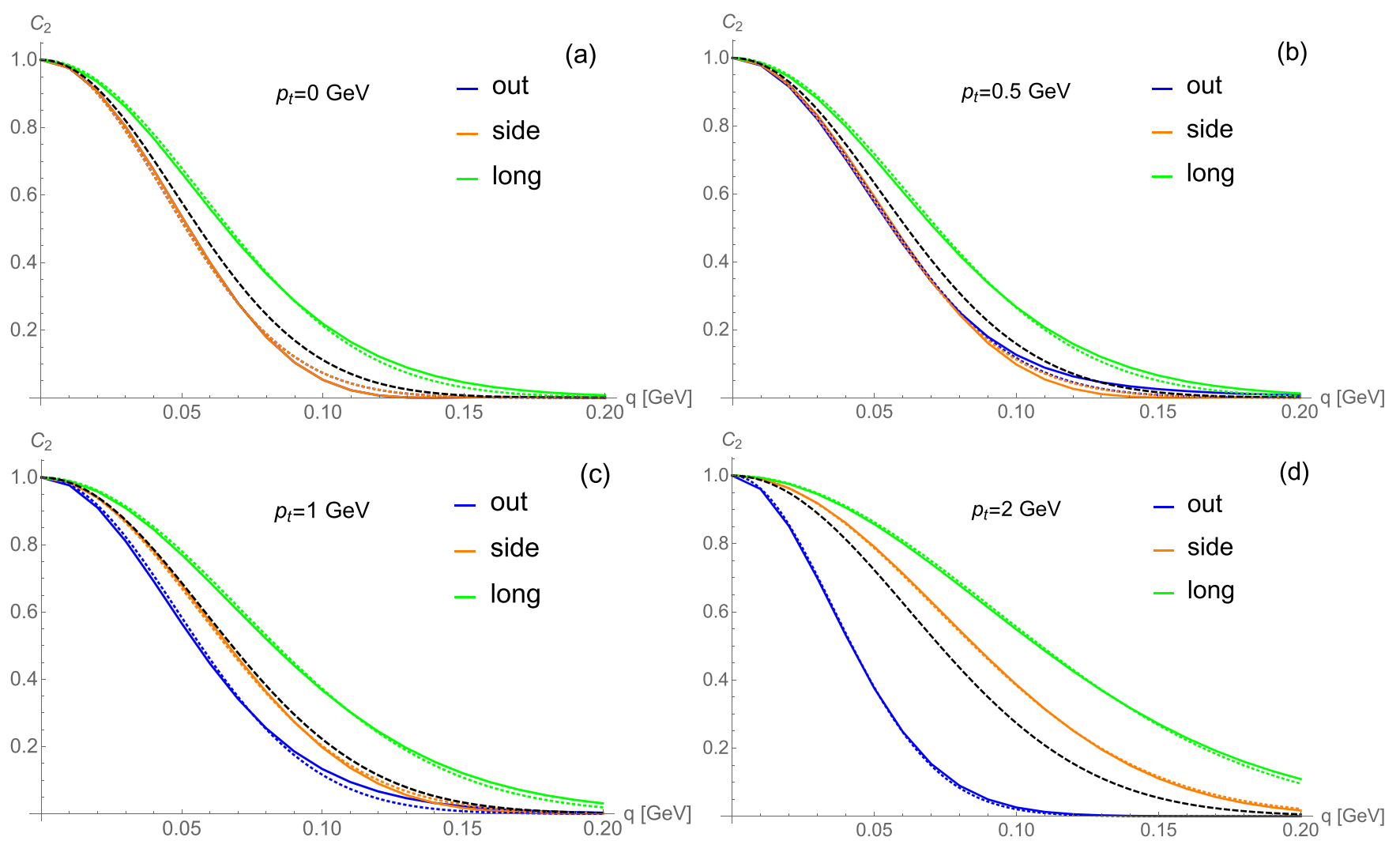

FIG. 9. The two-particle source $\mathcal{C}_{2}(p, \mathbf{q})$ calculated in the blast wave model and projected onto the out, side, long directions. Solid lines show the numerical result, while dotted lines show independent Gaussian fits to each projection. The black dashed line shows the 1D Gaussian source with $R_{\mathrm{inv}}=\left(R_{o} R_{s} R_{l}\right)^{\frac{1}{3}}$. The BWM parameters are chosen to mimic PbPb collisions at the LHC (see text). (a)-(d) $p_{t}=0,0.5,1,2 \mathrm{GeV}$.

and the azimuthal angle $\phi$. The position 4-vector is then $R^{\mu}=(\tau \cosh \eta, \rho \cos \phi, \rho \sin \phi, \tau \sinh \eta)$ and $d^{4} R=$ $d \tau \tau d \rho \rho d \eta d \phi$. The 4-momentum vector of a particle emitted at rapidity $Y$, azimuthal angle $\Phi$, and transverse momentum $p_{t}$, is $p^{\mu}=\left(m_{t} \cosh Y, p_{t} \cos \Phi, p_{t} \sinh \Phi, m_{t} \sinh Y\right)$, with $m_{t}=\sqrt{m^{2}+p_{t}^{2}}$. The single-particle emission function is parameterized using six parameters, $\tau_{0}, \Delta \tau, R_{0}, \beta_{S}, n, T$ (we neglect chemical potentials), as follows $[\theta(x)$ is the Heaviside step function]:

$$
\begin{aligned}
\tilde{S}_{p}(R)= & \sqrt{\frac{2}{\pi}} \frac{m_{t}}{m} \cosh (\eta-Y) J(\tau) \theta\left(R_{0}-\rho\right) e^{-\frac{p u}{T}} \\
u^{\mu}(R)= & \left(\cosh \eta \cosh \eta_{t}, \sinh \eta_{t} \cos \phi, \sinh \eta_{t} \sin \phi\right. \\
& \left.\sinh \eta \cosh \eta_{t}\right) \\
\eta_{t}(\rho)= & \tanh ^{-1}\left(\frac{\rho^{n}}{R_{0}^{n}} \beta_{S}\right) \\
J(\tau)= & \frac{1}{\sqrt{2 \pi} \Delta \tau} e^{-\frac{\left(\tau-\tau_{0}\right)^{2}}{2 \Delta \tau^{2}}} .
\end{aligned}
$$

With these definitions we can evaluate $\mathcal{C}_{2}(p, \mathbf{q})$ numerically via Eq. (25). In doing so, recall that we require $q=$ $(0, \mathbf{q})$ specified in the PRF, while the emission function $\tilde{S}_{p}(x)$ depends on space-time coordinates in the laboratory frame. Defining $b=p / m$, we can write $q^{\prime}$ in the laboratory frame as $q^{\prime 0}=\mathbf{b q}, \mathbf{q}^{\prime}=\mathbf{q}+\frac{\mathbf{b q}}{1+b^{0}} \mathbf{b}$ [15]. For simplicity, in the examples below we choose $p=\left(m_{t}, p_{t}, 0,0\right)$. With this choice $p u=m_{t} \cosh \eta \cosh \eta_{t}-p_{t} \sinh \eta_{t} \cos \phi$ and using the $q_{l}, q_{o}, q_{s}$ decomposition we have $q x=\frac{p_{t}}{m} q_{o} \tau \cosh \eta-$ $\frac{m_{t}}{m} q_{o} \rho \cos \phi-q_{s} \rho \sin \phi-q_{l} \tau \sinh \eta$. We consider separately the cases $\mathbf{q}=\left(q_{o}, 0,0\right), \mathbf{q}=\left(0, q_{s}, 0\right)$, and $\mathbf{q}=\left(0,0, q_{l}\right)$.

In Fig. 9 we plot $\mathcal{C}_{2}(p, \mathbf{q})$, projected onto the out, side, and long directions (solid blue, orange, and green, respectively). Independent Gaussian fits to each projection are shown by dotted lines. Black dashed line shows the 1D Gaussian source computed with $R_{\text {inv }}=\left(R_{o} R_{s} R_{l}\right)^{\frac{1}{3}}$. For definiteness we take $T=100 \mathrm{MeV}, n=1, \beta_{s}=0.5$ and $\tau_{0}=7 \mathrm{fm}, \Delta \tau=1.5 \mathrm{fm}$, $R_{0}=7 \mathrm{fm}$, chosen to roughly represent $\mathrm{PbPb}$ collisions [55]. Note that the phenomenological BWM is based on classical intuition and satisfies by construction, effectively, the density matrix factorization assumption. Thus the chaoticity parameters $\mathcal{N}_{2}=\mathcal{N}_{3}=1$ in this computation, and we are guaranteed that $\mathcal{C}_{2}(p, \mathbf{0})=1$.

The source depicted in Fig. 9 was chosen to resemble $\mathrm{PbPb}$ collisions. From the fits in the plot, the radii of homogeneity for this source at $p_{t}=0$, for example, are $R_{o}=R_{s} \approx 3.2 \mathrm{fm}$, $R_{l} \approx 2.5 \mathrm{fm}$, extended compared to the deuteron RMS radius $r_{\mathrm{rms}}=\sqrt{3 / 8} b_{d} \approx 2.1 \mathrm{fm}$. To see the size of the effect for smaller systems, e.g., $p p$ collisions, we recalculate $\mathcal{C}_{2}$ for different values of $R_{0}=\tau_{0}=2 \mathrm{fm}$, leading to $R_{o}=R_{s} \approx 0.9 \mathrm{fm}$, $R_{l} \approx 1 \mathrm{fm}$ at $p_{t}=0$. (The other BWM parameters are unchanged.) The results are shown in Fig. 10. 

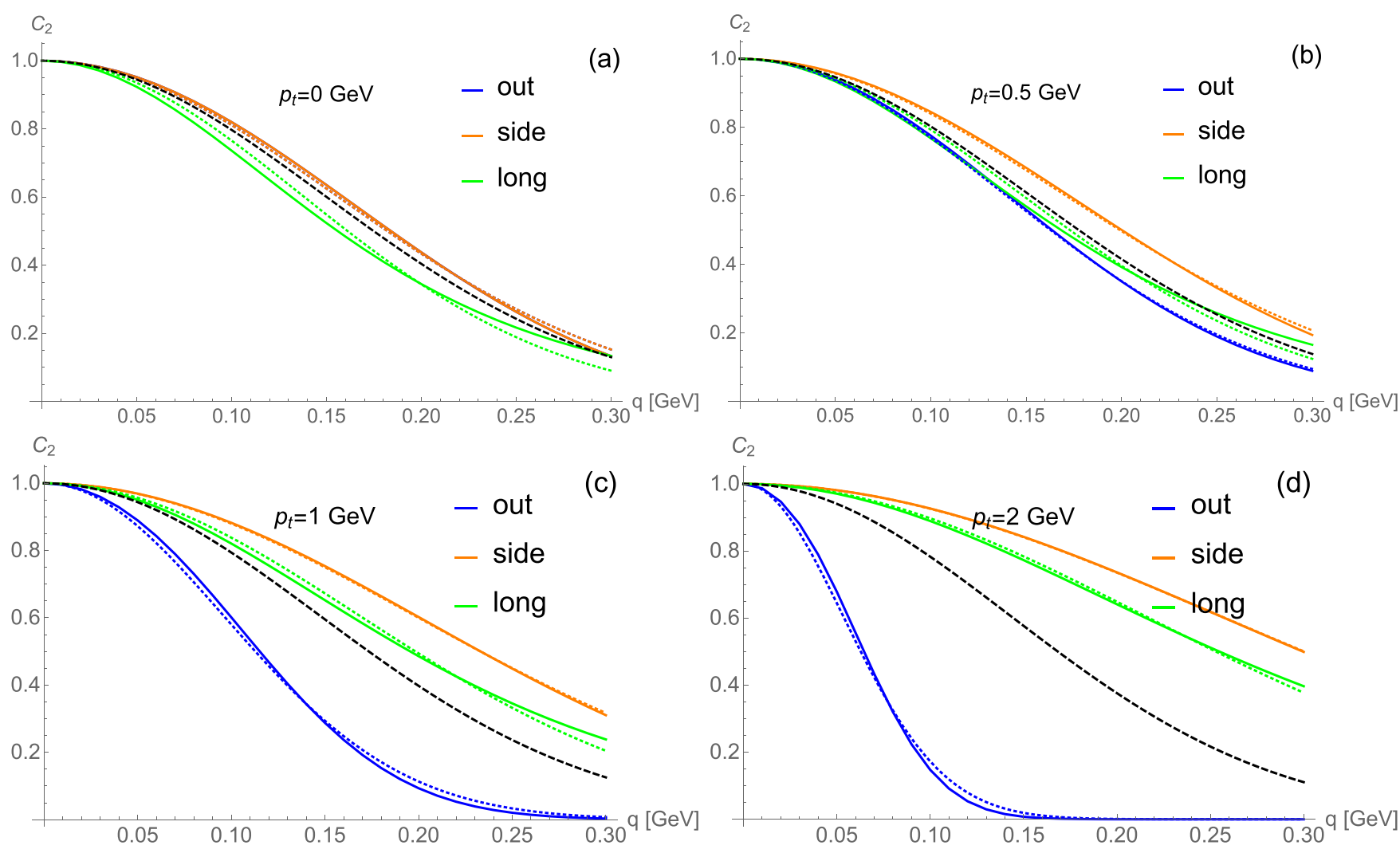

FIG. 10. Same as Fig. 9, with BWM model parameters chosen to mimic $p p$ collisions at the LHC.

Figures 9 and 10 demonstrate how unisotropic flow leads to an unisotropic source. Notably, the width along the out direction shows Lorentz contraction in the PRF at $p_{t}>0$. This perspective should also be taken into account in future studies that attempt to fit the details of $\mathcal{S}_{2}(\mathbf{r})$ to $O(10 \%)$ precision based on the 1D isotropic Gaussian model as in Ref. [20].

Our main interest is to check to what extent the deviation from 1D Gaussian source affects the coalescence calculation. Again from Figs. 9 and 10 (as well as from the discussion in Sec. A 2), we expect that a 3D Gaussian approximation may do a reasonably accurate job describing $\mathcal{C}_{2}$; thus we will use the 3D Gaussian parametrization as a standard for comparison. For a rough estimate of the error, incurred by using the 1D fit of Eq. (39) to describe a 3D source, we calculate $R_{o}, R_{s}, R_{l}$ in the BWM and define

$$
R_{\text {inv }}=\left(R_{o} R_{S} R_{l}\right)^{\frac{1}{3}}
$$

We can now compare the results of using Eq. (49) with $R_{o}=$ $R_{s}=R_{l} \rightarrow R_{\text {inv }}$ to the results of the original Eq. (49):

$$
\frac{\mathcal{B}_{2}^{1 D}}{\mathcal{B}_{2}^{3 D}} \approx \sqrt{\frac{\left(b_{d}^{2}+4 R_{l}^{2}\right)\left(b_{d}^{2}+4 R_{o}^{2}\right)\left(b_{d}^{2}+4 R_{s}^{2}\right)}{\left(b_{d}^{2}+4 R_{\text {inv }}^{2}\right)^{3}}} .
$$

Note that in both limits $b_{d} \rightarrow 0$ and $b_{d} \rightarrow \infty$ this correction factor is equal to 1 . The results are summarized in Table I.

We give a rough estimate of the correction to $\mathcal{B}_{3 \Lambda}$ in a similar way:

$$
\frac{\mathcal{B}_{3 \Lambda}^{1 D}}{\mathcal{B}_{3 \Lambda}^{3 D}} \approx \sqrt{\frac{\left(b_{p n}^{2}+2 R_{l}^{2}\right)\left(b_{\Lambda}^{2}+2 R_{l}^{2}\right)\left(b_{p n}^{2}+2 R_{o}^{2}\right)\left(b_{\Lambda}^{2}+2 R_{o}^{2}\right)\left(b_{p n}^{2}+2 R_{s}^{2}\right)\left(b_{\Lambda}^{2}+2 R_{s}^{2}\right)}{\left(b_{p n}^{2}+2 R_{\text {inv }}^{2}\right)^{3}\left(b_{\Lambda}^{2}+2 R \text { inv }^{2}\right)^{3}}}
$$

The calculation for $\mathcal{B}_{3}$ is the same up to $b_{p n} \rightarrow b_{\Lambda} \rightarrow b{ }^{\mathrm{He}}$. The results are also summarized in Table I.

We have also done a numerical calculation of the ratio $\mathcal{B}_{2}^{1 D} / \mathcal{B}_{2}^{3 D}$ using the Hulthen $\mathrm{D}$ wave function. In this exercise we calculated $\mathcal{B}_{2}^{3 D}$ from Eq. (15), comparing that with the result of Eq. (54). The results we find for $\mathrm{PbPb}$ and $p p$ and for all values of $p_{t}$ are numerically very close to those found in Table I using the Gaussian wave function.

The conclusion from Table I is that the 1D Gaussian parametrization tends to slightly overestimate the coalescence factor, in comparison to the more accurate $3 \mathrm{D}$ parametrization. The effect is more pronounced in small systems than 
TABLE I. The corrections of Eqs. (B6) and (B7), comparing between the coalescence factor obtained from a 1D and a 3D Gaussian fit to $\mathcal{C}_{2}$ computed in the BWM. We round the result to two significant digits.

\begin{tabular}{|c|c|c|c|c|c|c|c|}
\hline $\begin{array}{l}p_{t} \\
(\mathrm{GeV})\end{array}$ & $\begin{array}{c}m_{t} \\
(\mathrm{GeV})\end{array}$ & $\begin{array}{c}\mathcal{B}_{2}^{1 D} / \mathcal{B}_{2}^{3 D} \\
\mathrm{PbPb}\end{array}$ & $\begin{array}{c}\mathcal{B}_{2}^{1 D} / \mathcal{B}_{2}^{3 D} \\
p p\end{array}$ & $\begin{array}{c}\mathcal{B}_{3}^{1 D} / \mathcal{B}_{3}^{3 D} \\
\mathrm{PbPb}\end{array}$ & $\begin{array}{c}\mathcal{B}_{3}^{1 D} / \mathcal{B}_{3}^{3 D} \\
p p\end{array}$ & $\begin{array}{c}\mathcal{B}_{3 \Lambda}^{1 D} / \mathcal{B}_{3 \Lambda}^{3 D} \\
\mathrm{PbPb}\end{array}$ & $\begin{array}{c}\mathcal{B}_{3 \Lambda}^{1 D} / \mathcal{B}_{3 \Lambda}^{3 D} \\
p p\end{array}$ \\
\hline 0 & 0.94 & 1 & 1 & 1 & 1 & 1 & 1 \\
\hline 1 & 1.37 & 1 & 1.1 & 1 & 1.1 & 1 & 1.1 \\
\hline 2 & 2.2 & 1.1 & 1.3 & 1.2 & 1.9 & 1.2 & 1.5 \\
\hline
\end{tabular}

in $\mathrm{PbPb}$, and increases with increasing $p_{t}$. Using the $1 \mathrm{D}$ $R_{\text {inv }}$ parametrization results in $O(10 \%)$ error at $p_{t} \approx 1 \mathrm{GeV}$, rising to as much as a factor of 2 for ${ }^{3} \mathrm{He}$ at $p_{t}=2$ $\mathrm{GeV}$ in $p p$ collisions. It should be stressed that these conclusions are drawn from simple, model-dependent calculations in the BWM, and should only be taken as crude estimates of the theoretical uncertainty in the coalescence calculation.
[1] L. P. Csernai and J. I. Kapusta, Phys. Rep. 131, 223 (1986).

[2] A. Andronic, P. Braun-Munzinger, J. Stachel, and H. Stocker, Phys. Lett. B 697, 203 (2011).

[3] J. Cleymans, S. Kabana, I. Kraus, H. Oeschler, K. Redlich, and N. Sharma, Phys. Rev. C 84, 054916 (2011).

[4] A. Andronic, P. Braun-Munzinger, K. Redlich, and J. Stachel, Nature (London) 561, 321 (2018), 1710.09425.

[5] V. Vovchenko, B. Dönigus, and H. Stoecker, Phys. Lett. B 785, 171 (2018).

[6] V. Vovchenko, B. Dönigus, and H. Stoecker, Phys. Rev. C 100, 054906 (2019).

[7] S. Acharya et al. (ALICE Collaboration), Nucl. Phys. A 971, 1 (2018).

[8] E. Shuryak and J. M. Torres-Rincon, Phys. Rev. C 100, 024903 (2019).

[9] E. Shuryak and J. M. Torres-Rincon, Phys. Rev. C 101, 034914 (2020).

[10] M. Juric et al., Nucl. Phys. B 52, 1 (1973).

[11] M. A. Lisa, S. Pratt, R. Soltz, and U. Wiedemann, Annu. Rev. Nucl. Part. Sci. 55, 357 (2005).

[12] U. W. Heinz and B. V. Jacak, Annu. Rev. Nucl. Part. Sci. 49, 529 (1999).

[13] D. Oliinychenko, L.-G. Pang, H. Elfner, and V. Koch, Phys. Rev. C 99, 044907 (2019).

[14] R. Lednicky, Braz. J. Phys. 37, 939 (2007).

[15] R. Scheibl and U. W. Heinz, Phys. Rev. C 59, 1585 (1999).

[16] R. Lednicky and V. L. Lyuboshits, Yad. Fiz. 35, 1316 (1981) [Sov. J. Nucl. Phys. 35, 770 (1982)].

[17] S. V. Akkelin, R. Lednicky, and Y. M. Sinyukov, Phys. Rev. C 65, 064904 (2002).

[18] R. Lednicky, Phys. Part. Nucl. 40, 307 (2009).

[19] K. Blum and M. Takimoto, Phys. Rev. C 99, 044913 (2019).

[20] S. Acharya et al. (ALICE Collaboration), Phys. Lett. B 811, 135849 (2020).

[21] F. Hildenbrand and H. W. Hammer, Phys. Rev. C 100, 034002 (2019).

[22] Z. Zhang and C. M. Ko, Phys. Lett. B 780, 191 (2018).

[23] K.-J. Sun, C. M. Ko, and B. Dönigus, Phys. Lett. B 792, 132 (2019).

[24] F. Bellini and A. P. Kalweit, Phys. Rev. C 99, 054905 (2019).
[25] S. E. Koonin, Phys. Lett. B 70, 43 (1977).

[26] R. Bond, P. J. Johansen, S. E. Koonin, and S. Garpman, Phys. Lett. B 71, 43 (1977).

[27] H. Sato and K. Yazaki, Phys. Lett. B 98, 153 (1981).

[28] S. Mrowczynski, J. Phys. G 13, 1089 (1987).

[29] S. Mrowczynski, Phys. Lett. B 248, 459 (1990).

[30] S. Mrowczynski, Phys. Lett. B 277, 43 (1992).

[31] S. Mrowczynski, Phys. Lett. B 308, 216 (1993).

[32] S. Mrowczynski, Phys. Lett. B 345, 393 (1995).

[33] R. Maj and S. Mrowczynski, Phys. Rev. C 71, 044905 (2005).

[34] S. Mrowczynski, Acta Phys. Pol. B 48, 707 (2017).

[35] R. Maj and S. Mrowczynski, Phys. Rev. C 101, 014901 (2020).

[36] K. Blum, K. C. Y. Ng, R. Sato, and M. Takimoto, Phys. Rev. D 96, 103021 (2017).

[37] S. Pratt and M. B. Tsang, Phys. Rev. C 36, 2390 (1987).

[38] L. Martin, C. Gelbke, B. Erazmus, and R. Lednicky, Nucl. Phys. A 604, 69 (1996).

[39] S. S. Schweber, H. A. Bethe, and F. de Hoffmann, Mesons and Fields (Row, Peterson \& Co, Evanston, 1955).

[40] S. Pratt, Phys. Rev. C 56, 1095 (1997).

[41] L. D. Landau and E. Lifshits, Quantum Mechanics: NonRelativistic Theory, Course of Theoretical Physics Vol. 3 (Butterworth-Heinemann, Oxford, 1991).

[42] J. Adam et al. (ALICE Collaboration), Phys. Rev. C 92, 054908 (2015).

[43] S. Acharya et al. (ALICE Collaboration), Phys. Rev. C 99, 024001 (2019).

[44] D. Mihaylov, V. Mantovani Sarti, O. Arnold, L. Fabbietti, B. Hohlweger, and A. Mathis, Eur. Phys. J. C 78, 394 (2018).

[45] S. Mrowczynski and P. Slon, Acta Phys. Pol. B 51, 1739 (2020).

[46] D. Anchishkin, U. W. Heinz, and P. Renk, Phys. Rev. C 57, 1428 (1998).

[47] U. A. Wiedemann, B. Tomasik, and U. W. Heinz, Nucl. Phys. A 638, 475C (1998).

[48] S. Chapman, J. R. Nix, and U. W. Heinz, Phys. Rev. C 52, 2694 (1995).

[49] B. B. Abelev et al. (ALICE Collaboration), Phys. Lett. B 739, 139 (2014).

[50] U. A. Wiedemann and U. W. Heinz, Phys. Rev. C 56, 3265 (1997). 
[51] I. Sick, arXiv:1505.06924.

[52] L. Lamia, M. La Cognata, C. Spitaleri, B. Irgaziev, and R. G. Pizzone, Phys. Rev. C 85, 025805 (2012).

[53] J. G. Congleton, J. Phys. G 18, 339 (1992).

[54] B. Abelev et al. (ALICE Collaboration), Phys. Rev. D 87, 052016 (2013).

[55] J. Adam et al. (ALICE Collaboration), Phys. Rev. C 93, 024917 (2016).

[56] S. Acharya et al. (ALICE Collaboration), Phys. Rev. C 97, 024615 (2018).

[57] S. Acharya et al. (ALICE Collaboration), Phys. Rev. C 100, 024002 (2019).

[58] S. Acharya et al. (ALICE Collaboration), Phys. Rev. C 101, 044906 (2020).
[59] J. Adam et al. (ALICE Collaboration), Phys. Lett. B 754, 360 (2016).

[60] B. B. Abelev et al. (ALICE Collaboration), Phys. Rev. Lett. 111, 222301 (2013).

[61] B. Abelev et al. (ALICE Collaboration), Phys. Rev. C 88, 044910 (2013).

[62] S. Bazak and S. Mrowczynski, Eur. Phys. J. A 56, 193 (2020).

[63] Z. Citron et al., Working Group 5: Future Physics Opportunities for High-Density QCD at the LHC with Heavy-Ion and Proton Beams (CERN, Geneva, 2019), Vol. 7, pp. 1159-1410.

[64] J. Niedziela (ALICE Collaboration), EPJ Web Conf. 177, 04008 (2018).

[65] M. P. Szymanski, Ph.D. thesis, CICQM, Beijing, 2016 (unpublished). 